J. Nonlinear Sci. Vol. 17: pp. 369-397 (2007)

DOI: $10.1007 / \mathrm{s} 00332-006-0808-8$

\title{
Nonlinear
} Science

(C) 2007 Springer Science+Business Media, Inc.

\section{Spectral Stability of Traveling Water Waves: Analytic Dependence of the Spectrum}

\author{
David P. Nicholls \\ Department of Mathematics, Statistics, and Computer Science, \\ University of Illinois at Chicago, Chicago, IL 60607
}

Received September 13, 2006; revised manuscript accepted for publication March 8, 2007

Online publication May 31, 2007

Communicated by G. Iooss

\begin{abstract}
Summary. The motion of the free surface of an ideal fluid under the effects of gravity and capillarity arises in a number of problems of practical interest (e.g. open-ocean pollutant transport, deep-sea oil platform design, and the generation and propagation of tsunamis), and, consequently, the reliable and accurate numerical simulation of these "water waves" is of central importance. In a pair of recent papers the author, in collaboration with F. Reitich (Proc. Roy. Soc. Lond., A, 461(2057): 1283-1309 (2005); Euro. J. Mech. B/Fluids, 25(4): 406-424 (2006)), has developed a new, efficient, stable and highorder Boundary Perturbation scheme (the method of Transformed Field Expansions) for the robust numerical simulation of traveling solutions of the water wave equations. In this paper we extend this Boundary Perturbation technique to address the equally important topic of dynamic stability of these traveling wave forms. More specifically, we describe, and provide the theoretical justification for, a new numerical algorithm to compute the spectrum of the linearized water-wave problem as a function of a parameter, $\varepsilon$, meant to measure the amplitude of the traveling wave. In order to demonstrate the utility of this new method, we also present a sample calculation for two-dimensional waves in water of infinite depth subject to quite general two-dimensional perturbations.
\end{abstract}

\section{Introduction}

The motion of the surface of an ideal fluid under the influence of gravity and capillarity, known as the Water Wave Problem, is one of the oldest and most fundamental in fluid mechanics. Successful modeling techniques for this problem are important in applications such as open-ocean pollutant transport, deep-sea oil platform design, and the generation and propagation of tsunamis. In a pair of recent papers [NR05], [NR06], the author, in collaboration with F. Reitich, has developed a new, efficient, stable, and high-order Boundary Perturbation scheme (based upon rigorous mathematical analysis) 
for the robust numerical simulation of traveling solutions of the water wave equations. In this paper we extend this Boundary Perturbation technique (the method of Transformed Field Expansions) to address the equally important topic of dynamic stability of these traveling wave forms. More specifically we describe, and provide the theoretical justification for, a new numerical algorithm to compute the spectrum of the linearized water-wave problem as a function of a parameter, $\varepsilon$, meant to measure the amplitude of the traveling wave.

The question of stability of traveling water waves has received a great deal of attention, and a full account of the results known to date is well beyond the scope of this paper; we refer the reader to one of the excellent survey papers available on the subject, e.g. Dias \& Kharif [DK99]. These results can be classified not only by the type of basic traveling wave they consider but also by the class of permitted perturbations which may grow or decay from this equilibrium state. For example, the seminal work of Benjamin and Feir [BF67], Zakharov [Zak68], and Longuet-Higgins [LH78a], [LH78b] focused upon two-dimensional (one depth dimension and one propagation dimension) Stokes waves and the evolution of two-dimensional perturbations from these. By contrast, the calculations of Chen and Saffman [CS85] and MacKay and Saffman [MS86] focus upon three-dimensional perturbations of two-dimensional traveling waves, while Ioualalen et al., e.g. [IKR99], [IO02], consider three-dimensional perturbations of threedimensional patterns, the "short-crested waves." Another means of classification is the periodicity requirements of the traveling wave and/or perturbation: e.g. if the traveling wave is periodic, must the perturbation be periodic with respect to the same period cell? Finally, these results must specify a notion of stability: nonlinear, linear, or spectral? In this work we will consider traveling waves that can be generated by the algorithm of Nicholls and Reitich [NR05], [NR06] (e.g. periodic Stokes waves in two dimensions and periodic short-crested waves in three dimensions) and quite general Bloch periodic (or quasiperiodic) perturbations which need not have the same (or any) periodicity as the traveling wave. Additionally, the method considers the spectrum of the water wave problem linearized about these solutions and thus constitutes a study of spectral stability.

While there are still many open questions regarding the stability of traveling water waves, some general statements can be made. As Zakharov showed [Zak68], the water wave problem is a Hamiltonian system so that, among other things, the energy of the system is conserved. Furthermore, as the problem is time-reversible, the best stability that one can expect is weak stability (that small disturbances will remain small, i.e. neutral stability) and not strong stability (where small disturbances decay exponentially fast, i.e. asymptotic stability). In the context of a spectral stability analysis, this notion of weak stability is characterized by the spectrum of the linearization of the dynamical water-wave problem about a traveling solution being pure imaginary. As we shall see (Section 4.3), in the case of trivial traveling waves ("flat water"), this spectral stability analysis can be worked out explicitly and the entire spectrum is purely imaginary, though the issue of resonance does arise.

Of course we are primarily interested in nontrivial traveling profiles. A straightforward approach to determining stability is to linearize the water wave problem about an approximate traveling wave (computed, e.g., by [NR06]) and to approximate the spectrum via a numerical eigensolver. This linearization/eigensolve approach has been investigated by Ioualalen et al. [IKR99], [IO02] and is an active line of research being 
pursued by the author in collaboration with W. Craig using a stabilized numerical scheme. However, this method does ignore some of the information available to us regarding the traveling waves. In particular, it was shown in [CN00] (see also [RSh81], [NR05]) that traveling waves come in branches (two dimensions) or surfaces (three dimensions), which can be specified by a parameter $\varepsilon$ that is meant to represent wave height or wave slope. Furthermore, these branches/surfaces were shown to be jointly analytic with respect to not only the spatial variables but also the parameter $\varepsilon$ [NR05]. In the linearization/eigensolve procedure outlined above, this information is used solely to compute the basic traveling wave and information regarding the dependence of the spectrum upon $\varepsilon$ is lost. The point of view of this paper is that viewing the spectrum as an (analytic) function of the parameter $\varepsilon$ gives valuable insights into the nature of the onset of instability in this problem.

Adopting this latter point of view, one can imagine the spectrum "moving" smoothly as a function of the branch/surface parameter $\varepsilon$; this is guaranteed by our new theorem (Theorem 5.2) for generic choices of perturbation. The question now arises: Can spectrum on the imaginary axis (e.g. at $\varepsilon=0$ ) move into the right-half of the complex plane, resulting in instability of the base traveling wave? MacKay and Saffman [MS86], using the Hamiltonian structure of the water wave problem, showed that a necessary (though not sufficient) condition for eigenvalues to move off the imaginary axis is that they collide. (A sharper, though still not sufficient, condition can be given with their observation that this collision must occur between eigenvalues of opposite Krein signature [MS86].) This observation is important for us, as we propose to measure the strength of an instability by finding, for each configuration (i.e. choice of Bloch periodic perturbation), the value of $\varepsilon$ of the first eigenvalue collision.

It is well known that collisions can occur in the linear problem, i.e. there may be eigenvalues of multiplicity higher than one for $\varepsilon=0$. These instances of resonance have been the subject of a great deal of research [DK99] and represent an easily identified source of potential instability (though not all such collisions give rise to instability; see [MS86] Section 4 for traveling waves). Unfortunately, due to the nature of our scheme as it is currently formulated, we are unable to address configurations that feature these resonances (i.e. higher multiplicity of the eigenvalues). While this may, on the surface, appear to be a shortcoming of our approach, it is a reflection of the somewhat imprecise nature of our instability criterion (and that of [MS86], [IKR99], [IO02]): collision of eigenvalues. If we choose this as our test, then in a resonant configuration, instability already exists at $\varepsilon=0$ and more finely tuned techniques must be brought to bear upon the problem. However, if we are in a (generic) nonresonant configuration, then, as we shall see in Section 6, our new method can be used to give reliable and highly accurate estimates of the onset and strength of instabilities in traveling water waves.

The outline of the paper is as follows: In Section 2 we review the equations of motion of the water wave problem, while in Section 3 we recall a change of variables that we have found very useful in establishing analyticity results for boundary value and free boundary problems, as well as for their numerical simulation. Section 4 discusses the spectral stability analysis that we have in mind, where we show how one can incorporate the solutions of Nicholls and Reitich [NR06] into a robust, high-order numerical procedure to deduce information regarding their spectral stability. In Section 5 we give the analyticity result upon which our new numerical scheme is based, and in Section 6 we give some 
preliminary results for two-dimensional waves subject to two-dimensional perturbations. We conclude with remarks and future directions in Section 7, and in Appendix A we discuss existence and uniqueness aspects of the linear estimate which permits us to establish the analyticity theorem.

\section{Equations of Motion}

As mentioned in the introduction, we shall consider the motion of the free interface above an ideal (inviscid, irrotational, incompressible) fluid under the influence of capillarity and gravity. If the fluid occupies the $d$-dimensional $(d=2,3)$ domain

$$
S_{h, \eta}=\left\{x \in \mathbf{R}^{d-1} \mid-h<y<\eta(x, t)\right\},
$$

with mean depth $h$ and free surface $\eta=\eta(x, t)$, the equations of motion are known to be [Lam93]:

$$
\begin{array}{ll}
\Delta \varphi=0 & \text { in } S_{h, \eta}, \\
\partial_{y} \varphi=0 & \text { at } y=-h \\
\partial_{t} \eta+\nabla_{x} \varphi \cdot \nabla_{x} \eta-\partial_{y} \varphi=0 & \text { at } y=\eta \\
\partial_{t} \varphi+\frac{1}{2}|\nabla \varphi|^{2}+g \eta-\sigma \Delta_{x} \eta-\sigma H(\eta)=0 & \text { at } y=\eta,
\end{array}
$$

where $\varphi=\varphi(x, y, t)$ is the velocity potential $(\vec{v}=\nabla \varphi), H$ is the (modified) curvature:

$$
H(\eta):=\operatorname{div}_{x}\left[\frac{\nabla_{x} \eta}{\sqrt{1+\left|\nabla_{x} \eta\right|^{2}}}\right]-\Delta_{x} \eta,
$$

and $g$ and $\sigma$ are constants of gravity and capillarity, respectively. Water of infinite depth can be accommodated by replacing (1b) with

$$
\partial_{y} \varphi \rightarrow 0 \text { as } y \rightarrow-\infty,
$$

and these equations are also supplemented with initial conditions

$$
\eta(x, 0)=\eta_{0}(x), \quad \varphi\left(x, \eta_{0}(x), 0\right)=\xi_{0}(x),
$$

where it suffices (by elliptic theory) to specify $\varphi$ only at the surface. Boundary conditions must also be enforced to guarantee the existence of a unique solution; these can be periodic, Dirichlet, Neumann, or Robin, or can decay at infinity. For simplicity we will consider the classical periodic boundary conditions with respect to some lattice $\Gamma \subset \mathbf{R}^{d-1}$, i.e.

$$
\eta(x+\gamma, t)=\eta(x, t), \quad \varphi(x+\gamma, y, t)=\varphi(x, y, t) \quad \forall \gamma \in \Gamma ;
$$

this lattice generates the conjugate lattice of wavenumbers [Mie97],

$$
\Gamma^{\prime}:=\left\{k \in \mathbf{R}^{d} \mid k \cdot \gamma \in(2 \pi) \mathbf{Z}, \forall \gamma \in \Gamma\right\} .
$$


If we change coordinates to a reference frame translating uniformly with speed $c \in$ $\mathbf{R}^{d-1}$ in the $x$-direction, then the governing equations become (for water of finite depth)

$$
\begin{array}{ll}
\Delta \varphi=0 & \text { in } S_{h, \eta}, \\
\partial_{y} \varphi=0 & \text { at } y=-h, \\
\partial_{t} \eta+c \cdot \nabla_{x} \eta+\nabla_{x} \varphi \cdot \nabla_{x} \eta-\partial_{y} \varphi=0 & \text { at } y=\eta, \\
\partial_{t} \varphi+c \cdot \nabla_{x} \varphi+\frac{1}{2}|\nabla \varphi|^{2}+g \eta-\sigma \Delta_{x} \eta-\sigma H(\eta)=0 & \text { at } y=\eta, \\
\eta(x, 0)=\eta_{0}(x), & \\
\varphi\left(x, \eta_{0}(x), 0\right)=\xi_{0}(x), &
\end{array}
$$

again with periodic boundary conditions.

\subsection{A Transparent Boundary Condition}

We can greatly reduce the size of the problem domain, $S_{h, \eta}$, for (2) with the introduction of a "transparent boundary condition" [NR05], [NR06]. This boundary condition not only is important for computational considerations (significantly reducing the domain to be discretized), but also permits a uniform statement of the water wave problem over all depths including the infinite depth case. To begin we consider a hyperplane $y=-a$ such that $-h<-a<|\eta|_{L^{\infty}}$. We next consider the augmented version of (2)

$$
\begin{array}{ll}
\Delta v=0 & \text { in } S_{a, \eta} \\
\partial_{t} \eta+c \cdot \nabla_{x} \eta+\nabla_{x} v \cdot \nabla_{x} \eta-\partial_{y} v=0 & \text { at } y=\eta \\
\partial_{t} v+c \cdot \nabla_{x} v+\frac{1}{2}|\nabla v|^{2}+g \eta-\sigma \Delta_{x} \eta-\sigma H(\eta)=0 & \text { (3b) } \\
\eta(x, 0)=\eta_{0}(x) & \text { at } y=\eta \\
v\left(x, \eta_{0}(x), 0\right)=\xi_{0}(x) & \\
\partial_{y} v=\partial_{y} w & \text { at } y=-a \\
\Delta w=0 & -h<y<-a(3 \mathrm{c}) \\
v=w & \text { at } y=-a \\
\partial_{y} w=0 & \text { at } y=-h ;
\end{array}
$$

again, deep water can be simulated if (3i) is replaced by

$$
\partial_{y} w \rightarrow 0 \quad \text { as } y \rightarrow-\infty .
$$

The solutions of (3) are equivalent to those of (2) in that $\varphi=v$ in $S_{a, \eta}$ and $\varphi=w$ in $\{-h<y<-a\}$. We now gather (3g)-(3i):

$$
\begin{aligned}
& \Delta w=0, \quad-h<y<-a, \\
& w(x,-a)=\psi(x), \\
& \partial_{y} w(x,-h)=0,
\end{aligned}
$$


where we have denoted the generic Dirichlet data at $y=-a$ by $\psi(x)$, and we note that (4) has the exact solution:

$$
w(x, y, t)=\sum_{k \in \Gamma^{\prime}} \hat{\psi}_{k} \frac{\cosh (|k|(y+h))}{\cosh (|k|(h-a))} e^{i k \cdot x},
$$

where $\hat{\psi}_{k}$ is the $k$ th Fourier coefficient of $\psi(x)$. To close the system of equations ( $\left.3 \mathrm{a}\right)-(3 \mathrm{f})$ for $v$ we must, in (3f), compute $\partial_{y} w$ given $v(x,-a)=w(x,-a)=\psi(x)$, i.e. compute the Dirichlet-Neumann operator (DNO):

$$
T(a)[\psi]:=\partial_{y} w(x,-a)=\sum_{k \in \Gamma^{\prime}}|k| \tanh ((h-a)|k|) \hat{\psi}_{k} e^{i k \cdot x},
$$

which, if we define $D:=-i \nabla_{x}$, gives rise to the Fourier multiplier notation:

$$
T[\psi]:=|D| \tanh ((h-a)|D|)[\psi] .
$$

We note that this entire discussion can be repeated for the case of deep water $(h=\infty)$ using (3j); in this case $T=|D|$.

With this notation we can, upon setting $v=\varphi$, equivalently state (2) (for arbitrary depth) as

$$
\begin{array}{ll}
\Delta \varphi=0 & \text { in } S_{a, \eta}, \\
\partial_{y} \varphi-T[\varphi]=0 & \text { at } y=-a, \\
\partial_{t} \eta+c \cdot \nabla_{x} \eta+\nabla_{x} \varphi \cdot \nabla_{x} \eta-\partial_{y} \varphi=0 & \text { at } y=\eta, \\
\partial_{t} \varphi+c \cdot \nabla_{x} \varphi+\frac{1}{2}|\nabla \varphi|^{2}+g \eta-\sigma \Delta_{x} \eta-\sigma H(\eta)=0 & \text { at } y=\eta, \\
\eta(x, 0)=\eta_{0}(x), & \\
\varphi\left(x, \eta_{0}(x), 0\right)=\xi_{0}(x) . &
\end{array}
$$

\section{Change of Variables}

A useful technique in the analysis and simulation of free boundary problems such as the water wave problem, (5), is a change of variables. Typically, in two-dimensional simulations $(d=2)$ a conformal transformation can be effected that, among other things, preserves the homogeneous structure of (5a). However, this technique does not extend to three dimensions $(d=3)$, so instead we employ a "domain flattening" transformation, which has proven quite useful in establishing analyticity results for boundary value and free boundary problems [NR01a], [NR01b], [NR03], [NR04a], [NR04b],

$$
x^{\prime}=x, \quad y^{\prime}=a\left(\frac{y-\eta(x, t)}{a+\eta(x, t)}\right), \quad t^{\prime}=t .
$$

We note that these coordinates are by no means novel and are known as $\sigma$-coordinates in the atmospheric science community [Phi57] and the $C$-method in electromagnetics 
[CMR80], [LCGP99]. It is not hard to see that derivatives transform as

$$
\begin{aligned}
(a+\eta) \nabla_{x} & =(a+\eta) \nabla_{x^{\prime}}-\left(\nabla_{x^{\prime}} \eta\right)\left(a+y^{\prime}\right) \partial_{y^{\prime}} \\
(a+\eta) \operatorname{div}_{x} & =(a+\eta) \operatorname{div}_{x^{\prime}}-\left(\nabla_{x^{\prime}} \eta\right) \cdot\left(a+y^{\prime}\right) \partial_{y^{\prime}} \\
(a+\eta) \partial_{y} & =a \partial_{y^{\prime}} \\
(a+\eta) \partial_{t} & =(a+\eta) \partial_{t^{\prime}}-\left(\partial_{t^{\prime}} \eta\right)\left(a+y^{\prime}\right) \partial_{y^{\prime}}
\end{aligned}
$$

Setting

$$
u\left(x^{\prime}, y^{\prime}, t^{\prime}\right)=\varphi\left(x^{\prime},(a+\eta) y^{\prime} / a+\eta, t^{\prime}\right),
$$

we can rewrite $(5 a)$ as

$$
\Delta^{\prime} u=F\left(x^{\prime}, y^{\prime} ; \eta, u\right)
$$

where

$$
F=\operatorname{div}_{x^{\prime}}\left[F^{(1)}\right]+\partial_{y^{\prime}} F^{(2)}+F^{(3)},
$$

and

$$
\begin{aligned}
-a^{2} F^{(1)}= & 2 a \eta \nabla_{x^{\prime}} u+\eta^{2} \nabla_{x^{\prime}} u-a\left(a+y^{\prime}\right)\left(\nabla_{x^{\prime}} \eta\right) \partial_{y^{\prime}} u \\
& -\left(a+y^{\prime}\right) \eta\left(\nabla_{x^{\prime}} \eta\right) \partial_{y^{\prime}} u \\
-a^{2} F^{(2)}= & -a\left(a+y^{\prime}\right) \nabla_{x^{\prime}} \eta \cdot \nabla_{x^{\prime}} u-\left(a+y^{\prime}\right) \eta \nabla_{x^{\prime}} \eta \cdot \nabla_{x^{\prime}} u \\
& +\left(a+y^{\prime}\right)^{2}\left|\nabla_{x^{\prime}} \eta\right|^{2} \partial_{y^{\prime}} u \\
-a^{2} F^{(3)}= & -a \nabla_{x^{\prime}} \eta \cdot \nabla_{x^{\prime}} u-\eta \nabla_{x^{\prime}} \eta \cdot \nabla_{x^{\prime}} u+\left(a+y^{\prime}\right)\left|\nabla_{x^{\prime}} \eta\right|^{2} \partial_{y^{\prime}} u
\end{aligned}
$$

Equation (5b) transforms to

$$
\partial_{y} u\left(x^{\prime},-a\right)-T\left[u\left(x^{\prime},-a\right)\right]=J\left(x^{\prime} ; \eta, u\right),
$$

where

$$
a J=\eta T[u] .
$$

Equations (5c) and (5d) transform to

$$
\begin{array}{ll}
\partial_{t^{\prime}} \eta+c \cdot \nabla_{x^{\prime}} \eta-\partial_{y^{\prime}} u=Q\left(x^{\prime} ; \eta, u\right) & \text { at } y^{\prime}=0, \\
\partial_{t^{\prime}} u+c \cdot \nabla_{x^{\prime}} u+g \eta-\sigma \Delta_{x^{\prime}} \eta=R\left(x^{\prime} ; \eta, u\right) & \text { at } y^{\prime}=0,
\end{array}
$$

where

$$
-a Q=\eta \partial_{t^{\prime}} \eta+\eta\left(c \cdot \nabla_{x^{\prime}} \eta\right)+a\left(\nabla_{x^{\prime}} \eta\right) \cdot \nabla_{x^{\prime}} u+\eta\left(\nabla_{x^{\prime}} \eta\right) \cdot \nabla_{x^{\prime}} u-a\left|\nabla_{x^{\prime}} \eta\right|^{2} \partial_{y^{\prime}} u,
$$
and

$$
\begin{aligned}
-a^{2} R= & 2 a \eta \partial_{t^{\prime}} u+\eta^{2} \partial_{t^{\prime}} u-a^{2}\left(\partial_{t^{\prime}} \eta\right) \partial_{y^{\prime}} u-a \eta\left(\partial_{t^{\prime}} \eta\right) \partial_{y^{\prime}} u \\
& +2 a \eta\left(c \cdot \nabla_{x^{\prime}} u\right)+\eta^{2}\left(c \cdot \nabla_{x^{\prime}} u\right)-a^{2}\left(c \cdot \nabla_{x^{\prime}} \eta\right) \partial_{y^{\prime}} u-a \eta\left(c \cdot \nabla_{x^{\prime}} \eta\right) \partial_{y^{\prime}} u \\
& +\frac{1}{2} a^{2}\left|\nabla_{x^{\prime}} u\right|^{2}+a \eta\left|\nabla_{x^{\prime}} u\right|^{2}+\frac{1}{2} \eta^{2}\left|\nabla_{x^{\prime}} u\right|^{2}-a^{2} \nabla_{x^{\prime}} \eta \cdot \nabla_{x^{\prime}} u \partial_{y^{\prime}} u \\
& -a \eta \nabla_{x^{\prime}} \eta \cdot \nabla_{x^{\prime}} u \partial_{y^{\prime}} u+\frac{1}{2} a^{2}\left|\nabla_{x^{\prime}} \eta\right|^{2}\left(\partial_{y^{\prime}} u\right)^{2}+\frac{1}{2} a^{2}\left(\partial_{y^{\prime}} u\right)^{2}+2 a g \eta^{2}+g \eta^{3} \\
& -2 \sigma a \eta \Delta_{x^{\prime}} \eta-\sigma \eta^{2} \Delta_{x^{\prime}} \eta-\sigma a^{2} H(\eta)-2 \sigma a \eta H(\eta)-\sigma \eta^{2} H(\eta) .
\end{aligned}
$$


To summarize, after the change of variables (6), (5) can be restated (dropping primes) as

$$
\begin{aligned}
& \Delta u(x, y)=\operatorname{div}_{x}\left[F^{(1)}(x, y ; \eta, u)\right]+\partial_{y} F^{(2)}(x, y ; \eta, u) \\
& +F^{(3)}(x, y ; \eta, u), \quad \text { in } S_{a, 0}, \\
& \partial_{y} u(x,-a)-T[u(x,-a)]=J(x ; \eta, u), \\
& \partial_{t} \eta(x, t)+c \cdot \nabla_{x} \eta(x, t)-\partial_{y} u(x, 0, t)=Q(x ; \eta, u), \\
& \partial_{t} u(x, 0, t)+c \cdot \nabla_{x} u(x, 0, t)+g \eta(x, t)-\sigma \Delta_{x} \eta(x, t)=R(x ; \eta, u),
\end{aligned}
$$

where $F^{(1)}, F^{(2)}$, and $F^{(3)}$ are given in (7), and $J, Q$, and $R$ are defined in (8), (9), and (10), respectively. These are supplemented with the initial conditions (5e) and (5f), and periodic boundary conditions, each of which are trivially transformed.

\section{Spectral Stability Analysis}

To begin our spectral stability analysis, we consider a traveling wave solution of (1), i.e. a steady solution

$$
(\bar{u}, \bar{\eta}, \bar{c})=(\bar{u}(x, y), \bar{\eta}(x), \bar{c})
$$

of (11). With this we seek solutions to the full problem (11) in a frame traveling with speed $c=\bar{c}$, of the form

$$
u(x, y, t)=\bar{u}(x, y)+\delta \tilde{u}(x, y, t), \quad \eta(x, t)=\bar{\eta}(x)+\delta \tilde{\eta}(x, t),
$$

where $\delta \ll 1$ measures the magnitude of the small perturbation of the traveling state. Inserting this into (11) we find, to order $\mathcal{O}(\delta)$, that the perturbations $\tilde{u}$ and $\tilde{\eta}$ satisfy

$$
\begin{aligned}
& \Delta \tilde{u}=F_{u}(\bar{u}, \bar{\eta})[\tilde{u}]+F_{\eta}(\bar{u}, \bar{\eta})[\tilde{\eta}], \quad \text { in } S_{a, 0}, \\
& \partial_{y} \tilde{u}(x,-a)-T[\tilde{u}(x,-a)]=J_{u}(\bar{u}, \bar{\eta})[\tilde{u}]+J_{\eta}(\bar{u}, \bar{\eta})[\tilde{\eta}], \\
& \partial_{t} \tilde{\eta}(x, t)+c \cdot \nabla_{x} \tilde{\eta}(x, t)-\partial_{y} \tilde{u}(x, 0, t)=Q_{u}(\bar{u}, \bar{\eta})[\tilde{u}]+Q_{\eta}(\bar{u}, \bar{\eta})[\tilde{\eta}], \\
& \partial_{t} \tilde{u}(x, 0, t)+c \cdot \nabla_{x} \tilde{u}(x, 0, t)+g \tilde{\eta}(x, t)-\sigma \Delta_{x} \tilde{\eta}(x, t) \\
& \quad=R_{u}(\bar{u}, \bar{\eta})[\tilde{u}]+R_{\eta}(\bar{u}, \bar{\eta})[\tilde{\eta}],
\end{aligned}
$$

where $u$ and $\eta$ variations are denoted with subscripts,

$$
F_{u}=\operatorname{div}_{x}\left[F_{u}^{(1)}\right]+\partial_{y} F_{u}^{(2)}+F_{u}^{(3)}, \quad F_{\eta}=\operatorname{div}_{x}\left[F_{\eta}^{(1)}\right]+\partial_{y} F_{\eta}^{(2)}+F_{\eta}^{(3)},
$$

and

$$
\begin{aligned}
-a^{2} F_{u}^{(1)}[\tilde{u}]= & 2 a \bar{\eta} \nabla_{x} \tilde{u}+\bar{\eta}^{2} \nabla_{x} \tilde{u}-a(a+y)\left(\nabla_{x} \bar{\eta}\right) \partial_{y} \tilde{u}-(a+y) \bar{\eta}\left(\nabla_{x} \bar{\eta}\right) \partial_{y} \tilde{u} \\
-a^{2} F_{\eta}^{(1)}[\tilde{u}]= & 2 a \tilde{\eta} \nabla_{x} \bar{u}+2 \bar{\eta} \tilde{\eta} \nabla_{x} \bar{u}-a(a+y)\left(\nabla_{x} \tilde{\eta}\right) \partial_{y} \bar{u} \\
& -(a+y) \tilde{\eta}\left(\nabla_{x} \bar{\eta}\right) \partial_{y} \bar{u}-(a+y) \bar{\eta}\left(\nabla_{x} \tilde{\eta}\right) \partial_{y} \bar{u}
\end{aligned}
$$




$$
\begin{aligned}
-a^{2} F_{u}^{(2)}[\tilde{u}]= & -a(a+y) \nabla_{x} \bar{\eta} \cdot \nabla_{x} \tilde{u}-(a+y) \bar{\eta} \nabla_{x} \bar{\eta} \cdot \nabla_{x} \tilde{u} \\
& +(a+y)^{2}\left|\nabla_{x} \bar{\eta}\right|^{2} \partial_{y} \tilde{u} \\
-a^{2} F_{\eta}^{(2)}[\tilde{\eta}]= & -a(a+y) \nabla_{x} \tilde{\eta} \cdot \nabla_{x} \bar{u}-(a+y) \tilde{\eta} \nabla_{x} \bar{\eta} \cdot \nabla_{x} \bar{u}-(a+y) \bar{\eta} \nabla_{x} \tilde{\eta} \cdot \nabla_{x} \bar{u} \\
& +2(a+y)^{2} \nabla_{x} \bar{\eta} \cdot \nabla_{x} \tilde{\eta} \partial_{y} \bar{u} \\
-a^{2} F_{u}^{(3)}[\tilde{u}]= & -a \nabla_{x} \bar{\eta} \cdot \nabla_{x} \tilde{u}-\bar{\eta} \nabla_{x} \bar{\eta} \cdot \nabla_{x} \tilde{u}+(a+y)\left|\nabla_{x} \bar{\eta}\right|^{2} \partial_{y} \tilde{u} \\
-a^{2} F_{\eta}^{(3)}[\tilde{\eta}]= & -a \nabla_{x} \tilde{\eta} \cdot \nabla_{x} \bar{u}-\tilde{\eta} \nabla_{x} \bar{\eta} \cdot \nabla_{x} \bar{u}-\bar{\eta} \nabla_{x} \tilde{\eta} \cdot \nabla_{x} \bar{u} \\
& +2(a+y) \nabla_{x} \bar{\eta} \cdot \nabla_{x} \tilde{\eta} \partial_{y} \bar{u}
\end{aligned}
$$

Additionally,

$$
\begin{aligned}
& a J_{u}[\tilde{u}]=\bar{\eta} T[\tilde{u}] \\
& a J_{\eta}[\tilde{\eta}]=\tilde{\eta} T[\bar{u}],
\end{aligned}
$$

and

$$
\begin{aligned}
-a Q_{u}[\tilde{u}]= & a\left(\nabla_{x} \bar{\eta}\right) \cdot \nabla_{x} \tilde{u}+\bar{\eta}\left(\nabla_{x} \bar{\eta}\right) \cdot \nabla_{x} \tilde{u}-a\left|\nabla_{x} \bar{\eta}\right|^{2} \partial_{y} \tilde{u}, \\
-a Q_{\eta}[\tilde{\eta}]= & \bar{\eta} \partial_{t} \tilde{\eta}+\tilde{\eta}\left(c \cdot \nabla_{x} \bar{\eta}\right)+\bar{\eta}\left(c \cdot \nabla_{x} \tilde{\eta}\right)+a\left(\nabla_{x} \tilde{\eta}\right) \cdot \nabla_{x} \bar{u} \\
& +\tilde{\eta}\left(\nabla_{x} \bar{\eta}\right) \cdot \nabla_{x} \bar{u}+\bar{\eta}\left(\nabla_{x} \tilde{\eta}\right) \cdot \nabla_{x} \bar{u}-2 a \nabla_{x} \bar{\eta} \cdot \nabla_{x} \tilde{\eta} \partial_{y} \bar{u}
\end{aligned}
$$

and

$$
\begin{aligned}
-a^{2} R_{u}[\tilde{u}]= & 2 a \bar{\eta} \partial_{t} \tilde{u}+\bar{\eta}^{2} \partial_{t} \tilde{u} \\
& +2 a \bar{\eta}\left(c \cdot \nabla_{x} \tilde{u}\right)+\bar{\eta}^{2}\left(c \cdot \nabla_{x} \tilde{u}\right)-a^{2}\left(c \cdot \nabla_{x} \bar{\eta}\right) \partial_{y} \tilde{u}-a \bar{\eta}\left(c \cdot \nabla_{x} \bar{\eta}\right) \partial_{y} \tilde{u} \\
& +a^{2} \nabla_{x} \bar{u} \cdot \nabla_{x} \tilde{u}+2 a \bar{\eta} \nabla_{x} \bar{u} \cdot \nabla_{x} \tilde{u}+\bar{\eta}^{2} \nabla_{x} \bar{u} \cdot \nabla_{x} \tilde{u} \\
& -a^{2} \nabla_{x} \bar{\eta} \cdot \nabla_{x} \tilde{u} \partial_{y} \bar{u}-a^{2} \nabla_{x} \bar{\eta} \cdot \nabla_{x} \bar{u} \partial_{y} \tilde{u} \\
& -a \bar{\eta} \nabla_{x} \bar{\eta} \cdot \nabla_{x} \tilde{u} \partial_{y} \bar{u}-a \bar{\eta} \nabla_{x} \bar{\eta} \cdot \nabla_{x} \bar{u} \partial_{y} \tilde{u} \\
& +a^{2}\left|\nabla_{x^{\prime}} \bar{\eta}\right|^{2}\left(\partial_{y} \bar{u}\right)\left(\partial_{y} \tilde{u}\right)+a^{2}\left(\partial_{y} \bar{u}\right)\left(\partial_{y} \tilde{u}\right) \\
-a^{2} R_{\eta}[\tilde{\eta}]= & -a^{2}\left(\partial_{t} \tilde{\eta}\right) \partial_{y} \bar{u}-a \bar{\eta}\left(\partial_{t} \tilde{\eta}\right) \partial_{y} \bar{u} \\
& +2 a \tilde{\eta}\left(c \cdot \nabla_{x} \bar{u}\right)+2 \bar{\eta} \tilde{\eta}\left(c \cdot \nabla_{x} \bar{u}\right)-a^{2}\left(c \cdot \nabla_{x} \tilde{\eta}\right) \partial_{y} \bar{u} \\
& -a \tilde{\eta}\left(c \cdot \nabla_{x} \bar{\eta}\right) \partial_{y} \bar{u}-a \bar{\eta}\left(c \cdot \nabla_{x} \tilde{\eta}\right) \partial_{y} \bar{u} \\
& +a \tilde{\eta}\left|\nabla_{x} \bar{u}\right|^{2}+\bar{\eta} \tilde{\eta}\left|\nabla_{x} \bar{u}\right|^{2}-a^{2} \nabla_{x} \tilde{\eta} \cdot \nabla_{x} \bar{u} \partial_{y} \bar{u} \\
& -a \tilde{\eta} \nabla_{x} \bar{\eta} \cdot \nabla_{x} \bar{u} \partial_{y} \bar{u}-a \bar{\eta} \nabla_{x} \tilde{\eta} \cdot \nabla_{x} \bar{u} \partial_{y} \bar{u}+a^{2} \nabla_{x} \bar{\eta} \cdot \nabla_{x} \tilde{\eta}\left(\partial_{y} \bar{u}\right)^{2} \\
& +4 a g \bar{\eta} \tilde{\eta}+3 g \bar{\eta}^{2} \tilde{\eta}-2 \sigma a \tilde{\eta} \Delta_{x} \bar{\eta}-2 \sigma a \bar{\eta} \Delta_{x} \tilde{\eta}-2 \sigma \bar{\eta} \tilde{\eta} \Delta_{x} \bar{\eta}-\sigma \bar{\eta}^{2} \Delta_{x} \tilde{\eta} \\
& -\sigma a^{2} H_{\eta}(\bar{\eta})[\tilde{\eta}]-2 \sigma a \tilde{\eta} H(\bar{\eta})-2 \sigma a \bar{\eta} H_{\eta}(\bar{\eta})[\tilde{\eta}] \\
& -2 \sigma \bar{\eta} \tilde{\eta} H(\bar{\eta})-\sigma \bar{\eta}^{2} H_{\eta}(\bar{\eta})[\tilde{\eta}] .
\end{aligned}
$$

We now posit "spectral stability" forms for $\tilde{u}$ and $\tilde{\eta}$,

$$
\tilde{u}(x, y, t)=e^{\lambda t} v(x, y), \quad \tilde{\eta}(x, t)=e^{\lambda t} \zeta(x),
$$


so that the values of $\lambda$ determine the spectral stability of the traveling waves $(\bar{u}, \bar{\eta})$ : $\operatorname{Re}\{\lambda\}>0$ for any $\lambda$ implies spectral instability, $\operatorname{Re}\{\lambda\} \leq 0$ for all $\lambda$ implies weak spectral stability, and $\operatorname{Re}\{\lambda\}<0$ for all $\lambda$ implies strong spectral stability. Inserting these into (12) we find that $v$ and $\zeta$ satisfy

$$
\begin{aligned}
& \Delta v=F_{u}(\bar{u}, \bar{\eta})[v]+F_{\eta}(\bar{u}, \bar{\eta})[\zeta], \quad \text { in } S_{a, 0}, \\
& \partial_{y} v(x,-a)-T[v(x,-a)]=J_{u}(\bar{u}, \bar{\eta})[v]+J_{\eta}(\bar{u}, \bar{\eta})[\zeta], \\
& {\left[\lambda+c \cdot \nabla_{x}\right] \zeta(x)-\partial_{y} v(x, 0)=Q_{u}(\bar{u}, \bar{\eta}, \lambda)[v]+Q_{\eta}(\bar{u}, \bar{\eta}, \lambda)[\zeta],} \\
& {\left[\lambda+c \cdot \nabla_{x}\right] v(x, 0)+\left[g-\sigma \Delta_{x}\right] \zeta(x)} \\
& \quad=R_{u}(\bar{u}, \bar{\eta}, \lambda)[v]+R_{\eta}(\bar{u}, \bar{\eta}, \lambda)[\zeta] .
\end{aligned}
$$

In this case, $F_{u}, F_{\eta}, J_{u}$, and $J_{\eta}$ remain identical (with $(\tilde{u}, \tilde{\eta})$ replaced by $(v, \zeta)$ ). However,

$$
\begin{aligned}
-a Q_{u}[v]= & a\left(\nabla_{x} \bar{\eta}\right) \cdot \nabla_{x} v+\bar{\eta}\left(\nabla_{x} \bar{\eta}\right) \cdot \nabla_{x} v-a\left|\nabla_{x} \bar{\eta}\right|^{2} \partial_{y} v, \\
-a Q_{\eta}[\zeta]= & \lambda \bar{\eta} \zeta+\zeta\left(c \cdot \nabla_{x} \bar{\eta}\right)+\bar{\eta}\left(c \cdot \nabla_{x} \zeta\right)+a\left(\nabla_{x} \zeta\right) \cdot \nabla_{x} \bar{u} \\
& +\zeta\left(\nabla_{x} \bar{\eta}\right) \cdot \nabla_{x} \bar{u}+\bar{\eta}\left(\nabla_{x} \zeta\right) \cdot \nabla_{x} \bar{u}-2 a \nabla_{x} \bar{\eta} \cdot \nabla_{x} \zeta \partial_{y} \bar{u},
\end{aligned}
$$

and

$$
\begin{aligned}
-a^{2} R_{u}[v]= & 2 a \lambda \bar{\eta} v+\lambda \bar{\eta}^{2} v \\
& +2 a \bar{\eta}\left(c \cdot \nabla_{x} v\right)+\bar{\eta}^{2}\left(c \cdot \nabla_{x} v\right)-a^{2}\left(c \cdot \nabla_{x} \bar{\eta}\right) \partial_{y} v-a \bar{\eta}\left(c \cdot \nabla_{x} \bar{\eta}\right) \partial_{y} v \\
& +a^{2} \nabla_{x} \bar{u} \cdot \nabla_{x} v+2 a \bar{\eta} \nabla_{x} \bar{u} \cdot \nabla_{x} v+\bar{\eta}^{2} \nabla_{x} \bar{u} \cdot \nabla_{x} v \\
& -a^{2} \nabla_{x} \bar{\eta} \cdot \nabla_{x} v \partial_{y} \bar{u}-a^{2} \nabla_{x} \bar{\eta} \cdot \nabla_{x} \bar{u} \partial_{y} v \\
& -a \bar{\eta} \nabla_{x} \bar{\eta} \cdot \nabla_{x} v \partial_{y} \bar{u}-a \bar{\eta} \nabla_{x} \bar{\eta} \cdot \nabla_{x} \bar{u} \partial_{y} v \\
& +a^{2}\left|\nabla_{x} \bar{\eta}\right|^{2}\left(\partial_{y} \bar{u}\right)\left(\partial_{y} v\right)+a^{2}\left(\partial_{y} \bar{u}\right)\left(\partial_{y} v\right) \\
-a^{2} R_{\eta}[\zeta]= & -a^{2}(\lambda \zeta) \partial_{y} \bar{u}-a \bar{\eta}(\lambda \zeta) \partial_{y} \bar{u} \\
& +2 a \zeta\left(c \cdot \nabla_{x} \bar{u}\right)+2 \bar{\eta} \zeta\left(c \cdot \nabla_{x} \bar{u}\right)-a^{2}\left(c \cdot \nabla_{x} \zeta\right) \partial_{y} \bar{u} \\
& -a \zeta\left(c \cdot \nabla_{x} \bar{\eta}\right) \partial_{y} \bar{u}-a \bar{\eta}\left(c \cdot \nabla_{x} \zeta\right) \partial_{y} \bar{u} \\
& +a \zeta\left|\nabla_{x} \bar{u}\right|^{2}+\bar{\eta} \zeta\left|\nabla_{x} \bar{u}\right|^{2}-a^{2} \nabla_{x} \zeta \cdot \nabla_{x} \bar{u} \partial_{y} \bar{u} \\
& -a \zeta \nabla_{x} \bar{\eta} \cdot \nabla_{x} \bar{u} \partial_{y} \bar{u}-a \bar{\eta} \nabla_{x} \zeta \cdot \nabla_{x} \bar{u} \partial_{y} \bar{u}+a^{2} \nabla_{x} \bar{\eta} \cdot \nabla_{x} \zeta\left(\partial_{y} \bar{u}\right)^{2} \\
& +4 a g \bar{\eta} \zeta+3 g \bar{\eta}^{2} \zeta-2 \sigma a \zeta \Delta_{x} \bar{\eta}-2 \sigma a \bar{\eta} \Delta_{x} \zeta-2 \sigma \bar{\eta} \zeta \Delta_{x} \bar{\eta}-\sigma \bar{\eta}^{2} \Delta_{x} \zeta \\
& -\sigma a^{2} H_{\eta}(\bar{\eta})[\zeta]-2 \sigma a \zeta H(\bar{\eta})-2 \sigma a \bar{\eta} H_{\eta}(\bar{\eta})[\zeta] \\
& -2 \sigma \bar{\eta} \zeta H(\bar{\eta})-\sigma \bar{\eta}^{2} H_{\eta}(\bar{\eta})[\zeta] .
\end{aligned}
$$

\subsection{Bloch Theory}

The final specification we make for our spectral stability problem (17), which we now write abstractly as

$$
\mathcal{A}(x, y)[(v, \zeta)]=\lambda(v, \zeta)
$$


is the boundary conditions that $v$ and $\zeta$ must satisfy. For guidance we follow the "Generalized Principle of Reduced Instability" developed by Mielke [Mie97], which was inspired by the Bloch theory of Schrödinger equations with periodic potentials [RS78]. This method allows us to consider perturbations

$$
(v, \zeta) \in H^{2}(\mathbf{R} \times[-a, 0]) \times H^{2}(\mathbf{R}),
$$

or even

$$
(v, \zeta) \in H_{l u}^{2}(\mathbf{R} \times[-a, 0]) \times H_{l u}^{2}(\mathbf{R}),
$$

which are the Sobolev spaces built upon the class of uniformly local $L^{2}$ functions [Mie97]. The Generalized Principle of Reduced Instability distills this general setting to the study of the "Bloch waves," e.g.

$$
\zeta(x)=e^{i p \cdot x} Z(x),
$$

where $Z \in H^{2}(\mathbf{R} / \Gamma)$ and $\Gamma$ is the lattice of periodicity for the linear operator $\mathcal{A}=$ $\mathcal{A}(\bar{u}(x, \cdot), \bar{\eta}(x), \bar{c})$ in the $x$-variable.

Since $Z \in H^{2}(\mathbf{R} / \Gamma)$, it suffices to consider $p \in P\left(\Gamma^{\prime}\right)$, the fundamental cell of wavenumbers (e.g., if $\Gamma=(2 \pi) \mathbf{Z}$, then $\Gamma^{\prime}=\mathbf{Z}$, and $\left.P\left(\Gamma^{\prime}\right)=[0,1]\right)$. Thus we are left with the spectral problem [Mie97]

$$
\mathcal{A}_{p}[(V, Z)]=\lambda(V, Z),
$$

cf. (20), where $\mathcal{A}_{p}$ is the "Bloch operator"

$$
\mathcal{A}_{p}:=e^{-i p \cdot x} \mathcal{A}\left[e^{i p \cdot x}(V, Z)\right] .
$$

The crucial spectral identity (see [Mie97], Theorems 2.1 and A.4) is

$$
L^{2}-\operatorname{spec}(\mathcal{A})=L_{l u}^{2}-\operatorname{spec}(\mathcal{A})=\operatorname{closure}\left(\bigcup_{p \in P\left(\Gamma^{\prime}\right)} \operatorname{spec}\left(\mathcal{A}_{p}\right)\right) .
$$

Thus, we can obtain information about stability with respect to all of these perturbations by simply considering

$$
(V, Z) \in H^{2}((\mathbf{R} / \Gamma) \times[-a, 0]) \times H^{2}(\mathbf{R} / \Gamma)
$$

with $p \in P\left(\Gamma^{\prime}\right)$ appearing as a parameter [Mie97].

Mielke makes the remark that, while the sets that appear in (21) are equal, their types are quite different. For example, $L_{l u}^{2}-\operatorname{spec}(\mathcal{A})$ is point spectrum, while $L^{2}-\operatorname{spec}(\mathcal{A})$ may contain essential spectrum (see [Mie97], Remark 1 after Theorem 2.1). Importantly, each of the Bloch operators $\mathcal{A}_{p}$ is elliptic and defined on the bounded spatial domain $P(\Gamma) \times[-a, 0]$, where $P(\Gamma)$ is the fundamental period cell. Thus each $\mathcal{A}_{p}$ is a Fredholm operator of index zero with compact resolvent, implying that $\operatorname{spec}\left(\mathcal{A}_{p}\right)$ is point spectrum.

Before leaving this discussion, we point out that this Bloch analysis is equivalent to considering the linear operator $\mathcal{A}$ acting on "Bloch periodic" (quasiperiodic) functions $v(x, y)$ and $\zeta(x)$ that satisfy the "Bloch boundary conditions":

$$
v(x+\gamma, y)=e^{i p \cdot \gamma} v(x, y), \quad \zeta(x+\gamma)=e^{i p \cdot \gamma} \zeta(x), \quad \forall \gamma \in \Gamma .
$$


Notice that if $p$ is a rational number, then these functions will be periodic with respect to the lattice $\Gamma$. Such functions can be expanded as

$$
\begin{aligned}
\zeta(x) & =\sum_{k \in \Gamma^{\prime}} \hat{\zeta}_{k} e^{i(k+p) \cdot x}, \\
v(x, y) & =\sum_{k \in \Gamma^{\prime}} \hat{v}_{k}(y) e^{i(k+p) \cdot x},
\end{aligned}
$$

which we use extensively in the rest of this paper.

\subsection{Transformed Field Expansions}

In [NR05] we showed that solutions of (1) exist in branches (in two dimensions) and surfaces (in three dimensions), dependent on the perturbation parameter $\varepsilon \in \mathbf{R}^{d-1}$. For the sake of computational complexity (computing the full surface of solutions is quite expensive and a sequence of branches typically suffices) and simplicity, we focus upon a one-dimensional parameter $\varepsilon \in \mathbf{R}$ which gives rise to strongly convergent expansions of the form

$$
\bar{u}(x, y, \varepsilon)=\sum_{n=1}^{\infty} \bar{u}_{n}(x, y) \varepsilon^{n}, \quad \bar{\eta}(x, \varepsilon)=\sum_{n=1}^{\infty} \bar{\eta}_{n}(x) \varepsilon^{n}, \quad \bar{c}(\varepsilon)=\sum_{n=0}^{\infty} \bar{c}_{n} \varepsilon^{n} .
$$

We shall show in Section 5 that the linearized quantities $(v, \zeta, \lambda)$ also depend (strongly) analytically upon $\varepsilon$ and can similarly be expanded as

$$
v(x, y, \varepsilon)=\sum_{n=0}^{\infty} v_{n}(x, y) \varepsilon^{n}, \quad \zeta(x, \varepsilon)=\sum_{n=0}^{\infty} \zeta_{n}(x) \varepsilon^{n}, \quad \lambda(\varepsilon)=\sum_{n=0}^{\infty} \lambda_{n} \varepsilon^{n} .
$$

Inserting these expansions into (17), we realize the "Transformed Field Expansions" (TFE) recursions:

$$
\begin{aligned}
& \Delta v_{n}=\mathcal{F}_{n}, \quad \text { in } S_{a, 0}, \\
& \partial_{y} v_{n}(x,-a)-T\left[v_{n}(x,-a)\right]=\mathcal{J}_{n}, \\
& {\left[\lambda_{0}+c_{0} \cdot \nabla_{x}\right] \zeta_{n}(x)-\partial_{y} v_{n}(x, 0)=\mathcal{Q}_{n}-\lambda_{n} \zeta_{0}(x)-\sum_{l=1}^{n-1} \lambda_{n-l} \zeta_{l}(x),} \\
& {\left[\lambda_{0}+c_{0} \cdot \nabla_{x}\right] v_{n}(x, 0)+\left[g-\sigma \Delta_{x}\right] \zeta_{n}(x)} \\
& \quad=\mathcal{R}_{n}-\lambda_{n} v_{0}(x, 0)-\sum_{l=1}^{n-1} \lambda_{n-l} v_{l}(x, 0) .
\end{aligned}
$$

where

$$
\begin{aligned}
\mathcal{F} & :=F_{u}(\bar{u}, \bar{\eta})[v]+F_{\eta}(\bar{u}, \bar{\eta})[\zeta], \\
\mathcal{J} & :=J_{u}(\bar{u}, \bar{\eta})[v]+J_{\eta}(\bar{u}, \bar{\eta})[\zeta], \\
\mathcal{Q} & :=Q_{u}(\bar{u}, \bar{\eta})[v]+Q_{\eta}(\bar{u}, \bar{\eta})[\zeta]-\left(c-c_{0}\right) \nabla_{x} \zeta, \\
\mathcal{R} & :=R_{u}(\bar{u}, \bar{\eta})[v]+R_{\eta}(\bar{u}, \bar{\eta})[\zeta]-\left(c-c_{0}\right) \nabla_{x} v(x, 0),
\end{aligned}
$$


and

$$
(\mathcal{F}, \mathcal{J}, \mathcal{Q}, \mathcal{R})=\sum_{n=1}^{\infty}\left(\mathcal{F}_{n}, \mathcal{J}_{n}, \mathcal{Q}_{n}, \mathcal{R}_{n}\right) \varepsilon^{n}
$$

These TFE recursions not only permit the direct estimation of the quantities $\left\{v_{n}, \zeta_{n}, \lambda_{n}\right\}$ (Theorem 5.2), but also lead to a stable, high-order algorithm for the numerical study of the dynamic stability of traveling water waves (see Section 6).

\subsection{Zeroth Order}

Before proceeding to the estimation of the $\left\{v_{n}, \zeta_{n}, \lambda_{n}\right\}$ (Theorem 5.2), we focus upon the recursions, (24), at order zero. Besides providing the basis for our algorithm, this case also reproduces the classic stability calculation of the trivial solutions. In this case the right-hand side of (24) is zero, and we find that solutions of (24a) satisfying the Bloch boundary conditions have the form

$$
v_{0}(x, y)=\sum_{k}\left\{A_{k} e^{|k+p| y}+B_{k} e^{-|k+p| y}\right\} e^{i(k+p) \cdot x} .
$$

Equation (24b) demands that

$$
v_{0}(x, y)=\sum_{k} C_{k}\left\{\beta_{k} e^{|k+p| y}+\alpha_{k} e^{-|k+p| y}\right\} e^{i(k+p) \cdot x}
$$

where

$$
\begin{aligned}
& \alpha_{k}:=\{1-\tanh ((h-a)|k+p|)\} e^{-a|k+p|}, \\
& \beta_{k}:=\{1+\tanh ((h-a)|k+p|)\} e^{a|k+p|} .
\end{aligned}
$$

The boundary conditions imply that

$$
\zeta_{0}(x)=\sum_{k} \hat{\zeta}_{0, k} e^{i(k+p) \cdot x}
$$

while (24c) and (24d) give

$$
M_{k} w_{k}=0, \quad \forall k \in \Gamma^{\prime},
$$

for the matrix/vector pair

$$
M_{k}:=\left(\begin{array}{cc}
\lambda_{0}+i c_{0} \cdot(k+p) & -|k+p|\left(\beta_{k}-\alpha_{k}\right) \\
g+\sigma|k+p|^{2} & \left(\lambda_{0}+i c_{0} \cdot(k+p)\right)\left(\beta_{k}+\alpha_{k}\right)
\end{array}\right), \quad w_{k}:=\left(\begin{array}{c}
\hat{\zeta}_{0, k} \\
C_{k}
\end{array}\right) .
$$

Clearly (25) delivers only nontrivial solutions when the matrix $M_{k}$ is singular for some wavenumber $k \in \Gamma^{\prime}$; this occurs when the (scaled) determinant function,

$$
\begin{aligned}
\Lambda\left(k, p ; \lambda_{0}, c_{0}, g, h, \sigma\right):= & \frac{\left(\lambda_{0}+i c_{0} \cdot(k+p)\right)^{2}\left(\beta_{k}+\alpha_{k}\right)}{\left(g+\sigma|k+p|^{2}\right)|k+p|\left(\beta_{k}-\alpha_{k}\right)} \\
\beta_{k}+\alpha_{k} & \left(\lambda_{0}+i c_{0} \cdot(k+p)\right)^{2} \\
& +\left(g+\sigma|k+p|^{2}\right)|k+p| \tanh (h|k+p|),
\end{aligned}
$$


is equal to zero. Given that $\left(p, c_{0}, g, h, \sigma\right)$ are fixed, for a given $\kappa \in \Gamma^{\prime}$, we solve for the unique (up to sign) $\lambda_{0}^{s}(\kappa), s= \pm 1$, such that $\Lambda\left(\kappa, p ; \lambda_{0}^{s}, c_{0}, g, h, \sigma\right)=0$, i.e.

$$
\lambda_{0}^{s}(\kappa)=i\left[-c_{0} \cdot(\kappa+p)+s \omega_{\kappa+p}\right],
$$

where

$$
\omega_{k}^{2}:=\left(g+\sigma|k|^{2}\right)|k| \tanh (h|k|) .
$$

Since all of the $\lambda_{0}^{s}(\kappa)$ are purely imaginary, we recover the classical weak stability result for trivial traveling water waves. In this case we find solutions of (25) of the form

$$
\hat{\zeta}_{0, \kappa}=\tau|\kappa+p|\left(\beta_{\kappa}-\alpha_{\kappa}\right), \quad C_{\kappa}=\tau\left(\lambda_{0}+i c_{0} \cdot(\kappa+p)\right),
$$

for any $\tau \in \mathbf{C}$. In particular, taking $\tau=\rho e^{i \theta}$ for real $\rho$ and $\theta$, we find the solutions

$$
\begin{aligned}
& \zeta_{0}(x)=\rho|\kappa+p|\left(\beta_{\kappa}-\alpha_{\kappa}\right) e^{i((\kappa+p) \cdot x+\theta)}, \\
& v_{0}(x, y)=\rho\left(\lambda_{0}+i c_{0} \cdot(\kappa+p)\right)\left\{\beta_{\kappa} e^{|\kappa+p| y}+\alpha_{\kappa} e^{-|\kappa+p| y}\right\} e^{i((\kappa+p) \cdot x+\theta),}
\end{aligned}
$$

which, together with $\lambda_{0}=\lambda_{0}^{s}$, are the starting point for our algorithm.

We remark that since, at order zero, the linear operator associated with (24) is constant coefficient, a simple Fourier analysis could replace the discussion above. However, we have included the preceding calculation with general Bloch boundary conditions to set the stage for the analysis of Section 5.

\section{Analyticity}

In this section we provide the proof of the analyticity of the spectral data $(v, \zeta, \lambda)$ as a function of $\varepsilon$ provided that the parameter $p$ lies in a permissible set of nonresonant values (see (31)). Before presenting this, we briefly review some notation which is necessary to state our results.

Recalling the representations (22a) and (22b) for functions satisfying our Bloch boundary conditions, we define the Bloch $L^{2}$-based Sobolev spaces

$$
H_{p}^{s}:=\left\{u \in L^{2} \mid\|u\|_{H_{p}^{s}}<\infty\right\},
$$

$s \in \mathbf{Z}$, where

$$
\|u(x, y)\|_{H_{p}^{s}}^{2}:=\sum_{j=0}^{s} \sum_{k \in \Gamma^{\prime}}\langle k+p\rangle^{2 s-2 j} \int_{-a}^{0}\left|\partial_{y}^{j} \hat{u}_{k}(y)\right|^{2} d y,
$$

and $\langle k+p\rangle=\sqrt{1+(k+p)^{2}}$. Note that if $f=f(x)$ depends on $x$ alone, then the space $H_{p}^{s}$ can be defined for any $s \in \mathbf{R}$ by the norm

$$
\|f(x)\|_{H_{p}^{s}}^{2 s}:=\sum_{k \in \Gamma^{\prime}}\langle k+p\rangle^{2 s}\left|\hat{f}_{k}\right|^{2} .
$$

Of course, if we set $p=0$, then we recover the classical $L^{2}$-based Sobolev spaces $H^{s}$. Finally, we note the following algebra property for $H_{p}^{s}$ [Ada75]. 
Lemma 5.1. If $s>d / 2$, then $u \in H^{s}$ and $v \in H_{p}^{s}$ imply that the product $(u v) \in H_{p}^{s}$ and

$$
\|u v\|_{H_{p}^{s}} \leq M\|u\|_{H^{s}}\|v\|_{H_{p}^{s}}
$$

for a constant $M=M(d, s, p)$ depending only on $d, s$, and $p$.

We now turn to the restriction of our theory, namely that certain "resonant" configurations must be excluded. To see why this is the case, recall that, to start our algorithm, for a fixed set of parameters $\left(\Gamma, p, c_{0}, g, h, \sigma\right)$ we can select, for every $\kappa \in \Gamma^{\prime}$, a unique (up to sign) value $\lambda_{0}^{s}(\kappa)$ such that

$$
\Lambda\left(\kappa, p ; \lambda_{0}^{s}, c_{0}, g, h, \sigma\right)=0,
$$

cf. (27). As we shall see (Lemma 5.5), if $\Lambda(k, p) \neq 0$ for all $k \neq \kappa$, then (24) can be uniquely solved at any order $n$ and our theorem follows. Viewing $p$ as the adjustable "configuration parameter," we define the set of permissible, "nonresonant" $p$, for a fixed $\kappa$, by

$$
\Omega_{\kappa}(\Gamma):=\left\{p \in P\left(\Gamma^{\prime}\right) \mid \Lambda\left(k, p ; \lambda_{0}^{s}, c_{0}, g, \sigma\right) \neq 0 \forall k \in \Gamma^{\prime} \backslash\{\kappa\}\right\} .
$$

Regarding the resonant $p \notin \Omega_{\kappa}(\Gamma)$, we point out that while they do play an important role in the study of stability of capillary-gravity waves, they are not generic (the dimensionality of the set of resonant $p$ is strictly less than that of the set of nonresonant ones) and represent quite a different type of instability, i.e. one that is independent of the shape of the waveform. Ultimately, we view these resonant values as particularly strong instabilities (corresponding to collision of eigenvalues at $\varepsilon=0$ ), which require a more subtle analysis than our current formulation permits, and instead we focus upon the nonresonant configurations featuring a dependence upon the traveling waveform that we can detect.

We now present our main result.

Theorem 5.2. Given $\left(\kappa, \Gamma, p, c_{0}, g, h, \sigma\right)$ and any integer $s>d / 2$, provided that $p \in$ $\Omega_{\kappa}(\Gamma)$, if, for $n \geq 1$,

$$
\left\|\bar{u}_{n}\right\|_{H^{s+2}} \leq C_{0} \frac{B^{n}}{(n+1)^{r}}, \quad\left\|\bar{\eta}_{n}\right\|_{H^{s+5 / 2}} \leq C_{0} \frac{B^{n}}{(n+1)^{r}}, \quad\left|\bar{c}_{n-1}\right| \leq C_{0} \frac{B^{n-1}}{(n+1)^{r}},
$$

for any $r>d-1$ and some positive constants $C_{0}, B$, then for $n \geq 0$ the solutions $\left\{v_{n}, \zeta_{n}, \lambda_{n}\right\}$ of (24), starting with (28) and (30), satisfy

$$
\left\|v_{n}\right\|_{H_{p}^{s+2}} \leq K_{0} \frac{D^{n}}{(n+1)^{r}}, \quad\left\|\zeta_{n}\right\|_{H_{p}^{s+5 / 2}} \leq K_{0} \frac{D^{n}}{(n+1)^{r}}, \quad\left|\lambda_{n}\right| \leq K_{0} \frac{D^{n}}{(n+1)^{r}}
$$

for positive constants $K_{0}$ and $D$. In other words, provided that $p$ is a permissible Bloch period and the traveling waveform is parametrically analytic, then the spectral data is also parametrically analytic.

Remark 5.3. We note that Theorem 4.2 of [NR05] justifies the hypothesis (32), which, for $d>2$, requires that $\sigma>0$. 
As with other related work on analyticity properties of boundary value and free boundary problems [NR01a], [NR04a], [NR05], [HN05], [NT06], we work by induction in the perturbation order $n$. The key inductive lemma is now presented.

Lemma 5.4. Given an integer $s>d / 2$, suppose that, for $n \geq 1$,

$$
\left\|\bar{u}_{n}\right\|_{H^{s+2}} \leq C_{0} \frac{B^{n}}{(n+1)^{r}}, \quad\left\|\bar{\eta}_{n}\right\|_{H^{s+5 / 2}} \leq C_{0} \frac{B^{n}}{(n+1)^{r}}, \quad\left|\bar{c}_{n-1}\right| \leq C_{0} \frac{B^{n-1}}{(n+1)^{r}},
$$

for any $r>d-1$ and some positive constants $C_{0}, B$; and

$$
\left\|v_{n}\right\|_{H_{p}^{s+2}} \leq K_{0} \frac{D^{n}}{(n+1)^{r}}, \quad\left\|\zeta_{n}\right\|_{H_{p}^{s+5 / 2}} \leq K_{0} \frac{D^{n}}{(n+1)^{r}}, \quad\left|\lambda_{n}\right| \leq K_{0} \frac{D^{n}}{(n+1)^{r}},
$$

for all $n<N$, then

$$
\begin{aligned}
\left\|\mathcal{F}_{N}\right\|_{H_{p}^{s}} & \leq K_{0} K_{1} \frac{D^{N-1}}{(N+1)^{r}}, & \left\|\mathcal{J}_{N}\right\|_{H_{p}^{s+1 / 2}} \leq K_{0} K_{1} \frac{D^{N-1}}{(N+1)^{r}}, \\
\left\|\mathcal{Q}_{N}\right\|_{H_{p}^{s+1 / 2}} & \leq K_{0} K_{1} \frac{D^{N-1}}{(N+1)^{r}}, & \left\|\mathcal{R}_{N}\right\|_{H_{p}^{s+1 / 2}} \leq K_{0} K_{1} \frac{D^{N-1}}{(N+1)^{r}},
\end{aligned}
$$

for some positive constant $K_{1}$ and $D \geq q B$ where $q>1$.

Proof. For brevity we restrict our attention to one of the most challenging terms to be estimated on the right-hand side of (24),

$$
I_{N}:=-\frac{1}{a} \sum_{m=0}^{N-1} \sum_{l=0}^{m} \lambda_{l} \zeta_{m-l} \bar{\eta}_{N-m},
$$

which appears as the first term in the expansion of $\mathcal{Q}_{\eta, N}$, cf. (18). This term is particularly difficult because the unknowns appear in a nonlinear fashion (the product of $\lambda$ with $\zeta$ ). All other terms can be handled with a similar method using the techniques presented here.

Using the algebra property (Lemma 5.1), we estimate

$$
\begin{aligned}
\left\|I_{N}\right\|_{H_{p}^{s+1 / 2}} \leq & \frac{1}{a} \sum_{m=0}^{N-1} \sum_{l=0}^{m} M\left|\lambda_{l}\right|\left\|\zeta_{m-l}\right\|_{H_{p}^{s+1 / 2}}\left\|\bar{\eta}_{N-m}\right\|_{H^{s+1 / 2}} \\
\leq & K_{0} \frac{M K_{0} C_{0}}{a} \sum_{m=0}^{N-1}\left[\sum_{l=0}^{m} \frac{(m+1)^{r}}{(l+1)^{r}(m-l+1)^{r}}\right] \\
& \times D^{m} B^{N-m} \frac{1}{(m+1)^{r}(N-m+1)^{r}},
\end{aligned}
$$

where we have used (34) \& (35). If we define $\Sigma_{d-1}$ by

$$
\Sigma_{d-1}:=\sum_{l=0}^{m} \frac{(m+1)^{r}}{(l+1)^{r}(m-l+1)^{r}}<\infty,
$$


cf. [NR03] (proof of Lemma 7), then

$$
\begin{aligned}
\left\|I_{N}\right\|_{H_{p}^{s+1 / 2}} \leq & K_{0} \frac{M K_{0} C_{0} \Sigma_{d-1}}{a} B \frac{D^{N-1}}{(N+1)^{r}} \\
& \times\left[\sum_{m=0}^{N-1} \frac{(N+1)^{r}}{(m+1)^{r}(N-m+1)^{r}}\left(\frac{B}{D}\right)^{N-m-1}\right] \\
\leq & K_{0}\left(\frac{M K_{0} C_{0} \Sigma_{d-1}^{2}}{a} B\right) \frac{D^{N-1}}{(N+1)^{r}}
\end{aligned}
$$

provided that $B / D<1 / q$ for any $q>1$. In this case,

$$
\left\|I_{N}\right\|_{H_{p}^{s+1 / 2}} \leq K_{0} K_{1} \frac{D^{N-1}}{(N+1)^{r}}
$$

provided

$$
K_{1} \geq \frac{M K_{0} C_{0} \Sigma_{d-1}^{2}}{a} B
$$

The second lemma required is an elliptic estimate that is very similar in spirit to one presented in [NR05]. In fact, since the methods for establishing it are nearly identical to those presented in [NR05] (Lemma 4.5), we state the result without proof. However, the issue of solvability and uniqueness of solutions is sufficiently different that we comment on these issues in Appendix A.

Lemma 5.5. Given $(\kappa, \Gamma, p, c, g, h, \sigma)$, where $p \in \Omega_{\kappa}(\Gamma)$, and an integer $s \geq 0$, if $F \in H_{p}^{s}\left(S_{a, 0}\right), J \in H_{p}^{s+1 / 2}(P(\Gamma)), Q \in H_{p}^{s+1 / 2}(P(\Gamma))$, and $R \in H_{p}^{s+1 / 2}(P(\Gamma))$, then there exists a solution $(v, \zeta, \mu)$ of

$$
\begin{aligned}
& \Delta v(x, y)=F(x, y), \quad-a<y<0, \\
& \partial_{y} v(x,-a)-T[v(x,-a)]=J(x), \\
& {\left[\lambda+c \cdot \nabla_{x}\right] \zeta(x)-\partial_{y} v(x, 0)=Q(x)-\mu q_{\kappa} e^{i(\kappa+p) \cdot x},} \\
& {\left[\lambda+c \cdot \nabla_{x}\right] v(x, 0)+\left[g-\sigma \Delta_{x}\right] \zeta(x)=R(x)-\mu r_{\kappa} e^{i(\kappa+p) \cdot x},} \\
& v(x+\gamma, y)=e^{i p \cdot \gamma} v(x, y), \quad \forall \gamma \in \Gamma, \\
& \zeta(x+\gamma)=e^{i p \cdot \gamma} \zeta(x), \quad \forall \gamma \in \Gamma,
\end{aligned}
$$

where

$$
\begin{aligned}
& q_{\kappa}:=\tau|\kappa+p|\left(\beta_{\kappa}-\alpha_{\kappa}\right), \\
& r_{\kappa}:=\tau\left(\lambda_{0}+i c_{0} \cdot(\kappa+p)\right)\left(\beta_{\kappa}+\alpha_{\kappa}\right),
\end{aligned}
$$

cf. (30). In addition, if we require that

$$
\int_{P(\Gamma)} \zeta(x) e^{i \kappa \cdot x} d x=0
$$


then the solution is unique and satisfies

$$
\begin{aligned}
\|v\|_{H_{p}^{s+2}} & \leq C_{e}\left\{\|F\|_{H_{p}^{s}}+\|J\|_{H_{p}^{s+1 / 2}}+\|Q\|_{H_{p}^{s+1 / 2}}+\|R\|_{\left.H_{p}^{s+1 / 2}\right\}},\right. \\
\|\zeta\|_{H_{p}^{s+5 / 2}} & \leq C_{e}\left\{\|F\|_{H_{p}^{s}}+\|J\|_{H_{p}^{s+1 / 2}}+\|Q\|_{H_{p}^{s+1 / 2}}+\|R\|_{\left.H_{p}^{s+1 / 2}\right\}},\right. \\
|\mu| & \leq C_{e}\left\{\|F\|_{H_{p}^{s}}+\|J\|_{H_{p}^{s+1 / 2}}+\|Q\|_{H_{p}^{s+1 / 2}}+\|R\|_{H_{p}^{s+1 / 2}}\right\},
\end{aligned}
$$

for some constant $C_{e}=C_{e}(d, s, p)>0$.

We are now in a position to prove Theorem 5.2.

Proof. (Theorem 5.2) We work by induction and begin at order $n=0$. We recall that we have already chosen our solution at zeroth order, (28) and (30), and simply select

$$
K_{0}:=\max \left\{\left\|v_{0}\right\|_{H_{p}^{s+2}},\left\|\zeta_{0}\right\|_{H_{p}^{s+5 / 2}},\left|\lambda_{0}^{s}\right|\right\} .
$$

We now assume (33) for all $n<N$, and note that, due to Lemma 5.5, we can estimate

$$
\begin{aligned}
\left\|v_{N}\right\|_{H_{p}^{s+2}} & \leq C_{e}\left\{\left\|\mathcal{F}_{N}\right\|_{H_{p}^{s}}+\left\|\mathcal{J}_{N}\right\|_{H_{p}^{s+1 / 2}}+\left\|\mathcal{Q}_{N}\right\|_{H_{p}^{s+1 / 2}}+\left\|\mathcal{R}_{N}\right\|_{H_{p}^{s+1 / 2}}\right\}, \\
\left\|\zeta_{N}\right\|_{H_{p}^{s+5 / 2}} & \leq C_{e}\left\{\left\|\mathcal{F}_{N}\right\|_{H_{p}^{s}}+\left\|\mathcal{J}_{N}\right\|_{H_{p}^{s+1 / 2}}+\left\|\mathcal{Q}_{N}\right\|_{H_{p}^{s+1 / 2}}+\left\|\mathcal{R}_{N}\right\|_{H_{p}^{s+1 / 2}}\right\}, \\
\left|\lambda_{N}\right| & \leq C_{e}\left\{\left\|\mathcal{F}_{N}\right\|_{H_{p}^{s}}+\left\|\mathcal{J}_{N}\right\|_{H_{p}^{s+1 / 2}}+\left\|\mathcal{Q}_{N}\right\|_{H_{p}^{s+1 / 2}}+\left\|\mathcal{R}_{N}\right\|_{H_{p}^{s+1 / 2}}\right\} .
\end{aligned}
$$

From the inductive estimate, Lemma 5.4, we have that

$$
\begin{aligned}
\left\|v_{N}\right\|_{H_{p}^{s+2}} & \leq C_{e} 4 K_{0} K_{1} \frac{D^{N-1}}{(N+1)^{r}}, \\
\left\|\zeta_{N}\right\|_{H_{p}^{s+5 / 2}} & \leq C_{e} 4 K_{0} K_{1} \frac{D^{N-1}}{(N+1)^{r}}, \\
\left|\lambda_{N}\right| & \leq C_{e} 4 K_{0} K_{1} \frac{D^{N-1}}{(N+1)^{r}},
\end{aligned}
$$

and we are done, provided that $D \geq 4 C_{e} K_{1}$.

\section{Two-Dimensional Numerical Results}

In this section we describe a numerical implementation of the TFE recursions (24) and report upon a study of the stability of two-dimensional traveling gravity waves (Stokes waves) on deep water to general (Bloch periodic) two-dimensional disturbances. As we mentioned earlier, this configuration has been well studied in previous research (see the survey article of Dias and Kharif [DK99] and its extensive list of references for a sample), so we present these results here merely to indicate the capabilities of our new method.

\subsection{Numerical Method}

We now specialize to the case of deep water $(h=\infty)$, two dimensions $(d=2)$, and zero capillarity $(\sigma=0)$. Furthermore, we will nondimensionalize by choosing $g=1$ and 
$[0,2 \pi]$ as the fundamental period cell for our traveling wave; therefore, the wavenumbers $k$ are the integers. We recall from [NR05] that to find a branch of nontrivial traveling waves one must, for a chosen wavenumber $\bar{k}$, find a (zeroth order) velocity $\bar{c}_{0} \in \mathbf{R}^{d-1}$ such that $\Delta\left(\bar{c}_{0}, \bar{k}\right)=0$, where

$$
\Delta(c, k):=(c \cdot k)^{2}-\omega_{k}^{2},
$$

and $\omega_{k}$ is defined in (29). It is not hard to show that in the present configuration $(h=\infty$, $d=2, g=1, \sigma=0$ ) the velocity is

$$
\bar{c}_{0}=\sqrt{1 /|\bar{k}|}
$$

while the (linear) free surface has, in the traveling frame, the form

$$
\bar{\eta}_{1}(x)=C \cos (\bar{k} x) .
$$

Again, for the purposes of nondimensionalization, we set $\bar{k}=1$ so that $\bar{c}_{0}=1$.

Before continuing, let us consider the set of permissible configurations for our algorithm. For every $p \in P\left(\Gamma^{\prime}\right)$ we would like to study the evolution of $\lambda^{s}(\kappa ; \varepsilon)$ as a function of $\varepsilon$ for all $\kappa \in \Gamma^{\prime}$. Due to the resonance we described in Section 5, this is not possible for our algorithm as it is currently formulated, so we restrict our attention to the set of $p \in \Omega(\Gamma)$ where

$$
\Omega(\Gamma):=\bigcap_{\kappa \in \Gamma^{\prime}} \Omega_{\kappa}(\Gamma),
$$

cf. (31). To exhibit the size of $\Omega(\Gamma)$, in Figure 1 we plot, for 400 equally spaced $p \in[0,1]$, the values of

$$
\Lambda_{\min }^{s}(p):=\min _{k, \kappa \in I_{N_{x}}, k \neq \kappa} \Lambda\left(k, p ; \lambda_{0}^{s}(\kappa), c_{0}, g, h, \sigma\right), \quad s= \pm 1,
$$

where $I_{N_{x}}:=\left[-N_{x} / 2, N_{x} / 2-1\right]$, and $N_{x}=64$. While we see that the values $p=0,1 / 4$, $3 / 4$ are forbidden $(p=1$ will reproduce the case $p=0$ as the spectral data is periodic in $p$ [Mie97]), the set $\Omega(\Gamma)$ admits all other values of $p$.

Our numerical scheme is a Fourier(collocation)/Chebyshev(tau)/Taylor algorithm [GO77], [CHQZ88], [NR01b], [NR06] applied to the system of equations (24). Briefly, this amounts to approximating the unknowns $(v, \zeta, \lambda)$ by

$$
\begin{aligned}
& v^{\left(N, N_{x}, N_{y}\right)}(x, y, \varepsilon):=\sum_{n=0}^{N} \sum_{k=-N_{x} / 2}^{N_{x} / 2-1} \sum_{l=0}^{N_{y}} \hat{v}_{n}^{k, l} T_{l}\left(\frac{2 y+a}{a}\right) e^{i(k+p) x} \varepsilon^{n}, \\
& \zeta^{\left(N, N_{x}\right)}(x, \varepsilon):=\sum_{n=0}^{N} \sum_{k=-N_{x} / 2}^{N_{x} / 2-1} \hat{\zeta}_{n}^{k} e^{i(k+p) x} \varepsilon^{n}, \\
& \lambda^{(N)}(\varepsilon):=\sum_{n=0}^{N} \lambda_{n} \varepsilon^{n}
\end{aligned}
$$

where $T_{l}$ is the $l$ th Chebyshev polynomial. We determine the $\left(\hat{v}_{n}^{k, l}, \hat{\zeta}_{n}^{k}, \lambda_{n}\right)$ from (24) (together with the solvability and uniqueness considerations outlined in Appendix A) and 


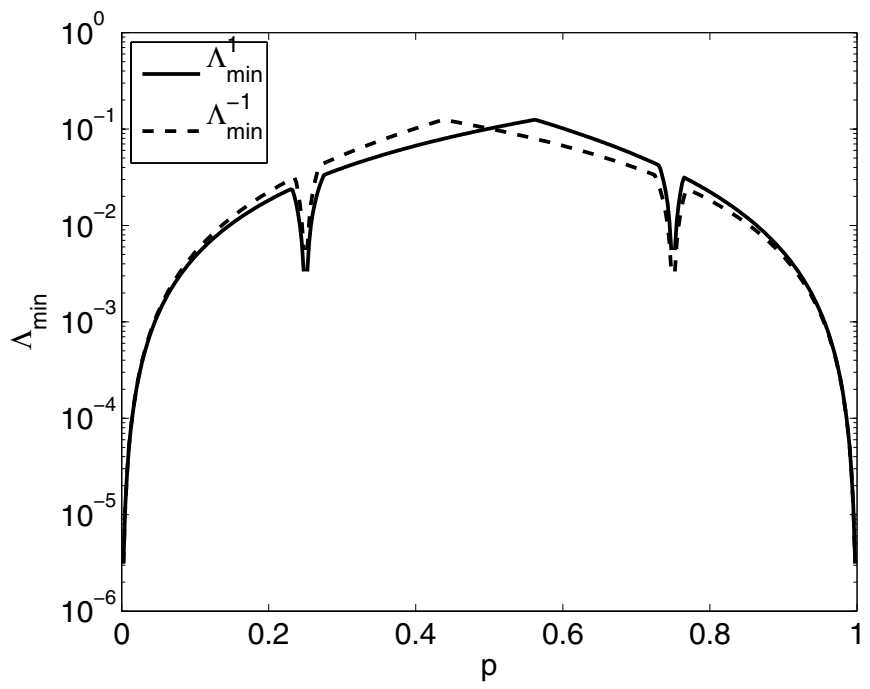

Fig. 1. Plot of $\Lambda_{\min }^{1}(p)$ and $\Lambda_{\min }^{-1}(p)$, cf. (40), for $N_{x}=64$ and 400 $p$ equally spaced in $[0,1]$.

the Fourier collocation ( $x$ variable) and Chebyshev tau ( $y$ variable) methods. Of course all of this depends on the faithful computation of the basic traveling wave $(\bar{\varphi}, \bar{\eta}, \bar{c})$, which we perform using the stable, high-order TFE method developed by Nicholls and Reitich [NR05], [NR06]. In the experiments of this section we have set $a=1 / 2, N=30$, $N_{x}=64$, and $N_{y}=32$ for the computation of both the traveling waveform $(\bar{\varphi}, \bar{\eta}, \bar{c})$ and the perturbation $(v, \zeta, \lambda)$.

Before proceeding, we note that there is an alternative method to sum the Taylor series (in $\varepsilon$ ) appearing in (41). It is well known that Padé approximants [BGM96] have remarkable properties of approximation for a large class of functions when applied to their Taylor series, not only within their disk of convergence, but also well outside (provided that there are no obstructing singularities present). In a practical implementation this generally means that a Padé approximation will not only converge faster where the Taylor series converges, but it may even converge for values of $\varepsilon$ where the Taylor series diverges. We have found this technique useful in a wide range of applications (e.g. [NR03], [NR04b]), and we use it here again.

\subsection{Computational Complexity}

Given our numerical approximation (41) to the problem (17), we can now comment on the computational complexity of our algorithm. We find both that the implementation of our new method is almost identical to that of Nicholls and Reitich [NR06] for traveling waves, and that the computational cost, for a fixed $p$, is the same:

$$
\mathcal{O}\left(N N_{x} \log \left(N_{x}\right) N_{y} \log \left(N_{y}\right)+N^{2} N_{x} N_{y}\right)
$$


the first term reflects the fast Fourier/Chebyshev solve at every perturbation order, while the second accounts for the time to assemble the right-hand sides $\mathcal{F}_{n}$, etc. (see (24)).

If we wish to compute $P$ Bloch periods $p$ then the total cost of this approach is

$$
\mathcal{O}\left(P N N_{x} \log \left(N_{x}\right) N_{y} \log \left(N_{y}\right)+P N^{2} N_{x} N_{y}\right)
$$

Given the $\left(N_{x} \times N_{y}\right)$ Fourier-Chebyshev coefficients $\hat{v}^{k, l}$, to compute the full spectrum for any given value of $\varepsilon$ we require only a Taylor (or Padé) summation of $N$ terms. Thus, for $P$-many periodicities, the cost of producing the spectrum for a particular nonlinear wave (a fixed $\varepsilon$ ) is

$$
\mathcal{O}\left(P N N_{x} N_{y}\right)
$$

From this we realize that the total cost for the full spectrum for $P$-many Bloch periodicities and $M$-many nonlinear waves is given by

$$
\mathcal{O}\left(P N N_{x} \log \left(N_{x}\right) N_{y} \log \left(N_{y}\right)+P N^{2} N_{x} N_{y}+M P N N_{x} N_{y}\right) .
$$

Notice that the computation of the spectral data is a post-processing step and that the parameter $M$ does not appear in the base cost of our algorithm. Note also that if the transparent boundary parameter $a$ is chosen small, then $N_{y}$ need not be chosen excessively large. If the parameters $N_{x}, N_{y}$, and $N$ are of comparable size, say $N$, and if $M \gg N$ (a finely resolved evolution of the spectrum), then the cost of our method is dominated by $\mathcal{O}\left(M P N^{3}\right)$.

We can now contrast this with alternative approaches, in particular the linearization/ eigensolve approach mentioned in the introduction. For this the dominant cost is the eigensolve, which has computational complexity $\mathcal{O}\left(L^{3}\right)$ if the linearization matrix has dimension $(L \times L)$ [GVL96]. The size of the linearization matrix associated with (17) is $L=N_{x} N_{y}$, so that the cost, for $P$-many Bloch periodicities, is

$$
\mathcal{O}\left(P\left(N_{x} N_{y}\right)^{3}\right)
$$

Importantly, this must be completely recomputed for each nonlinear wave, so that the cost of computing the full spectrum for $M$-many nonlinear waves is

$$
\mathcal{O}\left(M P\left(N_{x} N_{y}\right)^{3}\right)
$$

if, again, $N_{x}$ and $N_{y}$ are of comparable size, say $N$, then the total complexity is $\mathcal{O}\left(M P N^{6}\right)$ which is clearly disadvantaged when compared with our new approach, (42). We should note that the method of Ioualalen et al. [IKR99], [IO02] features matrices of size $\left(N_{x} \times N_{x}\right)$ so that the total cost of their algorithm is

$$
\mathcal{O}\left(M P N_{x}^{3}\right)
$$

which compares more favorably with the complexity of our new method (42). However, we point out that not only may $N_{y}$ be chosen quite small in our method, but the method of Ioualalen et al. is quite unstable (see the calculations of [NR06]), calling their very reliability into question. Furthermore, if all that is desired are the growth rates, $\lambda$, then our new approach will merely have complexity $\mathcal{O}(M P N)$ (once the Fourier/Chebyshev/Taylor 


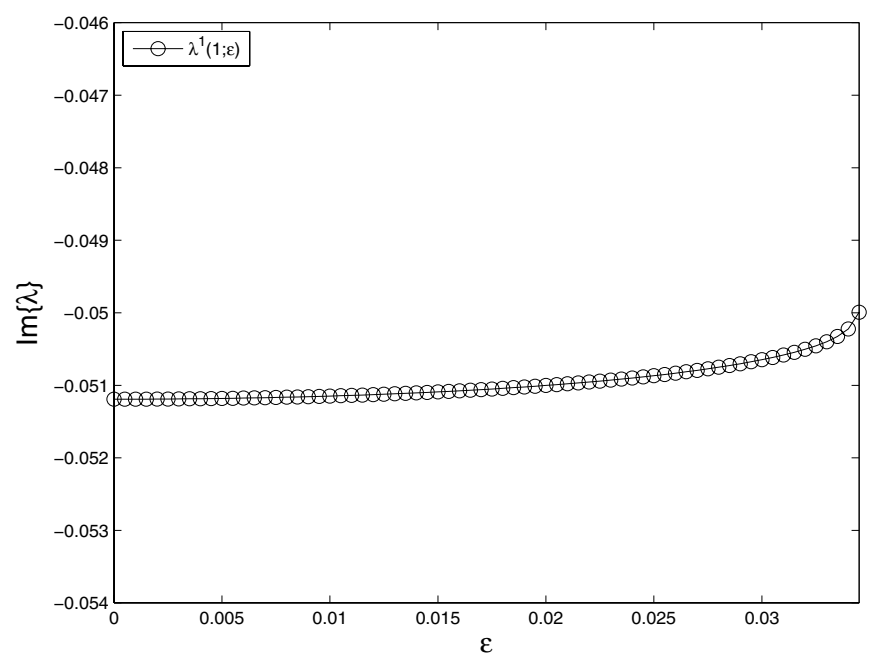

Fig. 2. Plot of $\operatorname{Im}\left\{\lambda^{1}(1 ; \varepsilon)\right\}$ for $N_{x}=64, N_{y}=32$, and $N=30$.

series are computed), while the cost of the linearization/eigensolve method remains the same.

Finally, in three dimensions each of these cost estimates remains the same save that $N_{x}$ must be replaced by $N_{1} \times N_{2}$ where $N_{j}$ is the number of collocation points in the $x_{j}$ direction. For our new method this multiplies the cost of our method by $N_{2} \log \left(N_{2}\right)$, while the linearization/eigensolve method has complexity multiplied by $N_{2}^{3}$, which renders it completely uncompetitive.

\subsection{Numerical Results}

To display the capabilities of our method, we begin by focusing on a particular choice of $p=1 / 10 \in \Omega(\Gamma)$, and perturbation wavenumber $\kappa=1$. In Figure 2 we display a plot of $\operatorname{Im}\left\{\lambda^{1}(1 ; \varepsilon)\right\}$ as a function of $\varepsilon$; we recall that, at $\varepsilon=0$, this eigenvalue has the form

$$
\lambda_{0}^{1}(1)=i\left[-c_{0}(1+0.1)+\omega_{1+0.1}\right] \approx-0.05119 i ;
$$

see (28). In this plot we see the smooth evolution of this eigenvalue as a function of $\varepsilon$ in the upward (imaginary) direction. Furthermore, throughout most values of the parameter $\varepsilon$ displayed, we feel that this calculation is highly resolved, as indicated in Figure 3. In this figure we display a plot of the difference between curves of $\operatorname{Im}\left\{\lambda^{1}(1 ; \varepsilon)\right\}$ for values of $N=12,18$, and 24 and that of the most resolved curve (appearing in Figure 2) with $N=30$. For values of $\varepsilon$ up to 0.005 it appears that full double-precision accuracy can be achieved with $N=12$ terms in the Taylor expansion, while $\varepsilon \leq 0.01$ and $\varepsilon \leq 0.015$ can be completely resolved with $N=18$ and $N=24$ terms, respectively.

One could imagine that, by simply increasing the perturbation order $N$ (and correspondingly increasing $N_{x}$ and $N_{y}$ to ensure high-order resolution), quite large values of $\varepsilon$ could be computed. However, as we display in Figure 4, there is a fundamental obstruction: collision of eigenvalues. In this figure we plot two curves, $\lambda^{1}(1 ; \varepsilon)$ and $\lambda^{-1}(-1 ; \varepsilon)$, 


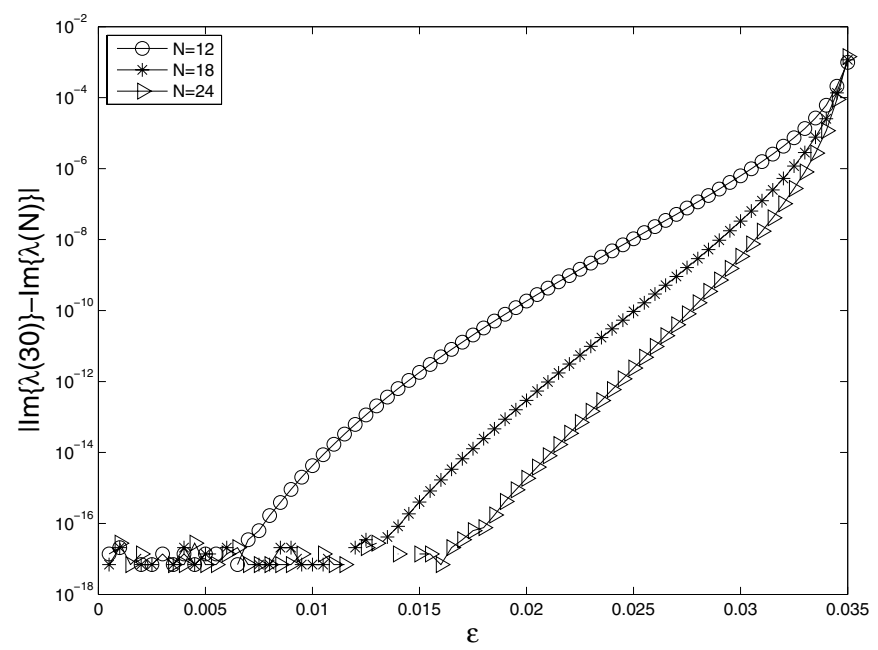

Fig. 3. Plot of $\left|\operatorname{Im}\left\{\lambda^{1}(1 ; \varepsilon, N)\right\}-\operatorname{Im}\left\{\lambda^{1}(1 ; \varepsilon, 30)\right\}\right|$ for $N_{x}=64$, $N_{y}=32$, and $N=12,18,24$.

which emanate, at $\varepsilon=0$, from the eigenvalues

$$
\begin{aligned}
& \lambda_{0}^{1}(1)=i\left[-c_{0}(1+0.1)+\omega_{1+0.1}\right] \approx-0.05119 i, \\
& \lambda_{0}^{-1}(-1)=i\left[-c_{0}(-1+0.1)-\omega_{-1+0.1}\right] \approx-0.04868 i,
\end{aligned}
$$

respectively. These are followed in a smooth fashion by our algorithm, with each progressing in the opposite (imaginary) direction towards each other. At a value of approximately $\varepsilon \approx 0.034$, these curves collide and we find that our computation rapidly loses

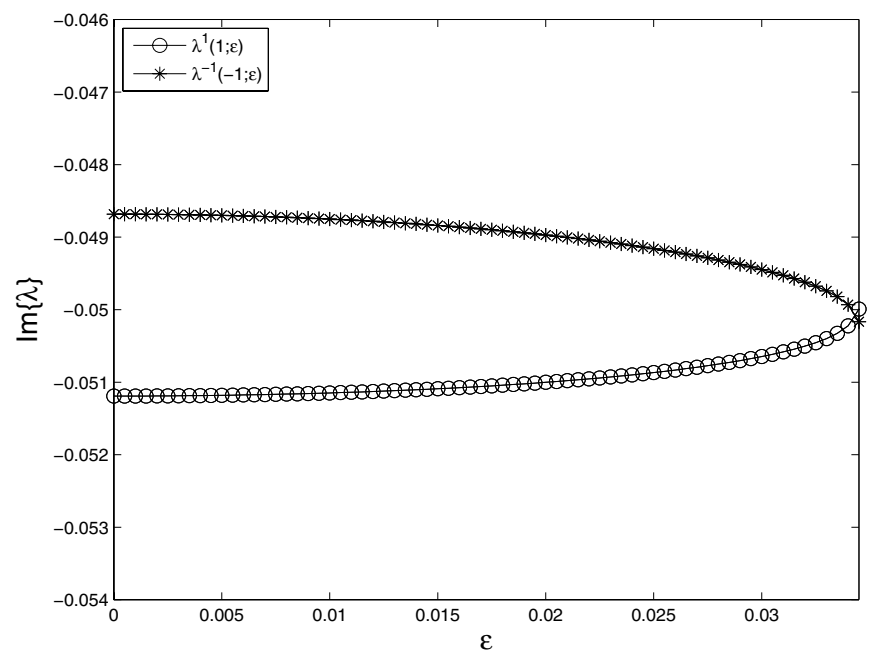

Fig. 4. Plot of $\lambda^{1}(1 ; \varepsilon)$ and $\lambda^{-1}(-1 ; \varepsilon)$ for $N_{x}=64, N_{y}=32$, and $N=30$. 
Table 1. Values of $\varepsilon_{\text {cross }}$ and $\operatorname{Im}\left\{\lambda\left(\varepsilon_{\text {cross }}\right)\right\}$ as $N$ is varied through values from 4 to 30 .

\begin{tabular}{rll}
\hline$N$ & $\varepsilon_{\text {cross }}$ & $\operatorname{Im}\left\{\lambda\left(\varepsilon_{\text {cross }}\right)\right\}$ \\
\hline 4 & 0.03939 & -0.050078 \\
6 & 0.03695 & -0.050111 \\
8 & 0.03593 & -0.050078 \\
10 & 0.03538 & -0.050095 \\
12 & 0.03507 & -0.050077 \\
14 & 0.03485 & -0.050091 \\
16 & 0.03472 & -0.050076 \\
18 & 0.03461 & -0.050093 \\
20 & 0.03452 & -0.050068 \\
22 & 0.03455 & -0.050063 \\
24 & 0.03448 & -0.050090 \\
26 & 0.03443 & -0.050078 \\
28 & 0.03440 & -0.050083 \\
30 & 0.03438 & -0.050080 \\
\hline
\end{tabular}

convergence. In fact, this is the first collision of eigenvalues in this configuration (choice of $p$ ), and we speculate that at this value of $\varepsilon$, say $\varepsilon_{\text {cross }}$, the expansions (23) cease to be convergent. Furthermore, since our technique of Padé approximation ceases to be useful beyond this point, we further theorize that there is a pole on the real axes of the complex $\varepsilon$-plane at $\varepsilon=\varepsilon_{\text {cross }}$.

In an attempt to build confidence in our computation of this "crossing point" we show, in Table 1, the numerical values of $\varepsilon_{\text {cross }}$ as the perturbation order $N$ is varied from 4 to 30. Additionally, we include the imaginary part of the eigenvalue where this crossing takes place. We point out that by $N=30$ we have convergence of $\varepsilon_{\text {cross }}$ to within a tenth of a percent, while $\operatorname{Im}\left\{\lambda\left(\varepsilon_{\text {cross }}\right)\right\}$ has converged to within a hundredth of a percent.

With confidence in our ability to compute "first crossing" values for the $\lambda$ as a function of $\varepsilon$ for a particular choice of $p$, we present in Figure 5 a plot of $\varepsilon_{\text {cross }}$ for a selection of $p$ representing the entire range $[0,1]$. This plot indicates the "strength" of the instability at each $p$ in the sense that larger $\varepsilon_{\text {cross }}$ indicates that larger (and more nonlinear) waves are still (weakly) stable. Furthermore, we can identify a value of $\varepsilon$, say $\varepsilon_{\text {crit }} \approx 0.0508$, for which sufficiently nonlinear $\left(\varepsilon>\varepsilon_{\text {crit }}\right)$ waves are unstable to perturbations of any Bloch period. Finally, in Figure 6 we display the values of the imaginary part of the eigenvalue $\lambda$ at the first crossing $\operatorname{Im}\left\{\lambda\left(\varepsilon_{\text {cross }}\right)\right\}$, and in Figure 7 we combine these last two plots (after a rescaling) to elucidate how discontinuities in the value of $\varepsilon_{\text {cross }}$ as a function of $p$ can be explained by the transition among "first cross" values of $\lambda$.

\section{Conclusions and Future Directions}

In this paper we have devised a novel method to investigate the stability of traveling water waves in two and three dimensions. We provide a theoretical justification for this algorithm via a rigorous analyticity theorem, and furthermore, the recursions used 


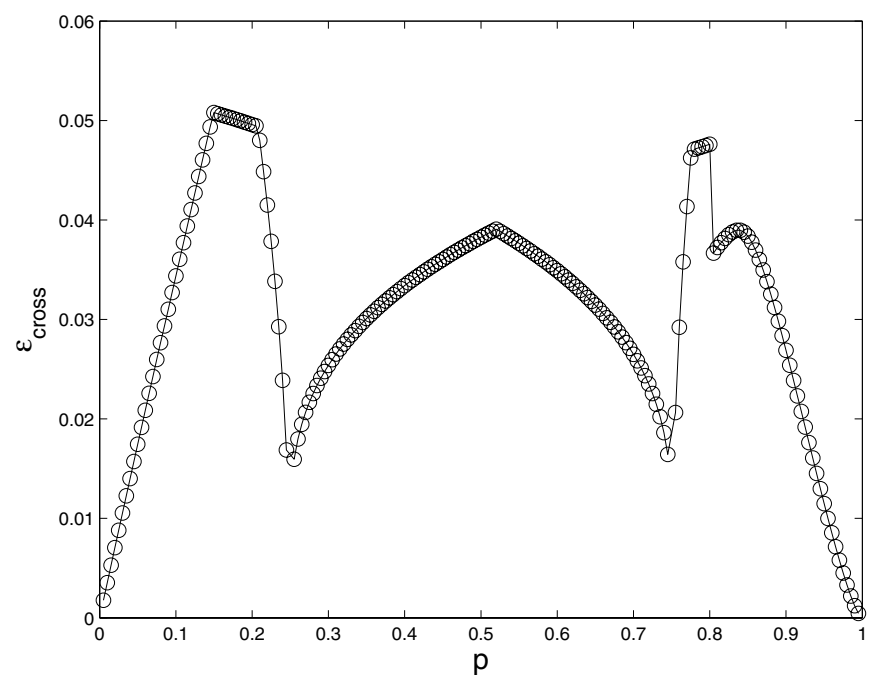

Fig. 5. Plot of "first crossing" $\varepsilon_{\text {cross }}$ versus $p$ for $N_{x}=64, N_{y}=32$, and $N=30$.

to establish this result translate directly into a numerical procedure for stability computations. A two-dimensional implementation was used to investigate the stability of two-dimensional gravity water waves on deep water to general two-dimensional perturbations. Clearly, the first future direction for this work is to extend our computations to three dimensions to consider the stability of "short-crested waves" to general threedimensional perturbations. Another interesting direction of future research would be to

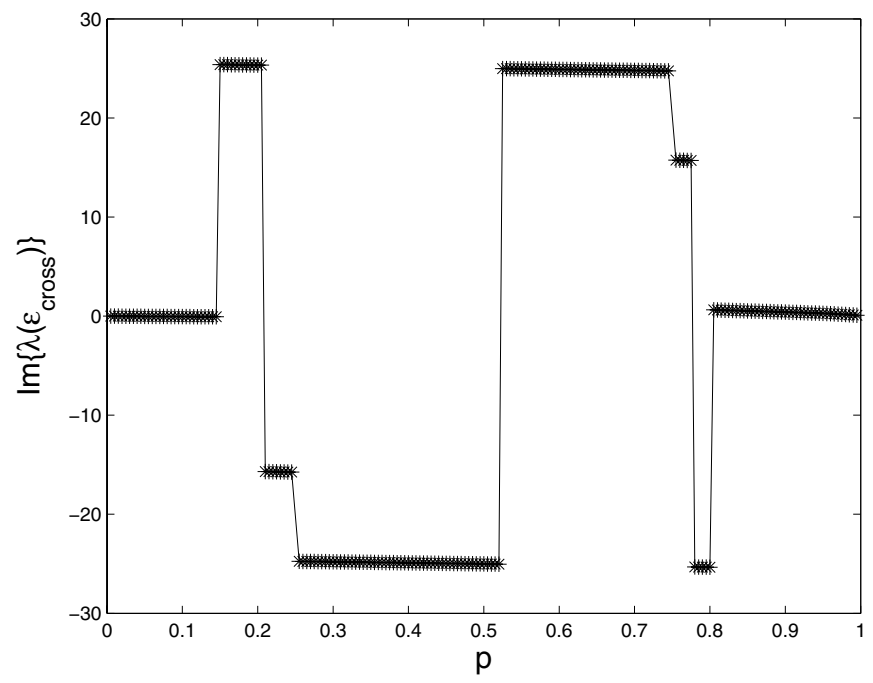

Fig. 6. Plot of the imaginary part of the "first crossing eigenvalue" $\lambda\left(\varepsilon_{\text {cross }}\right)$ versus $p$ for $N_{x}=64, N_{y}=32$, and $N=30$. 


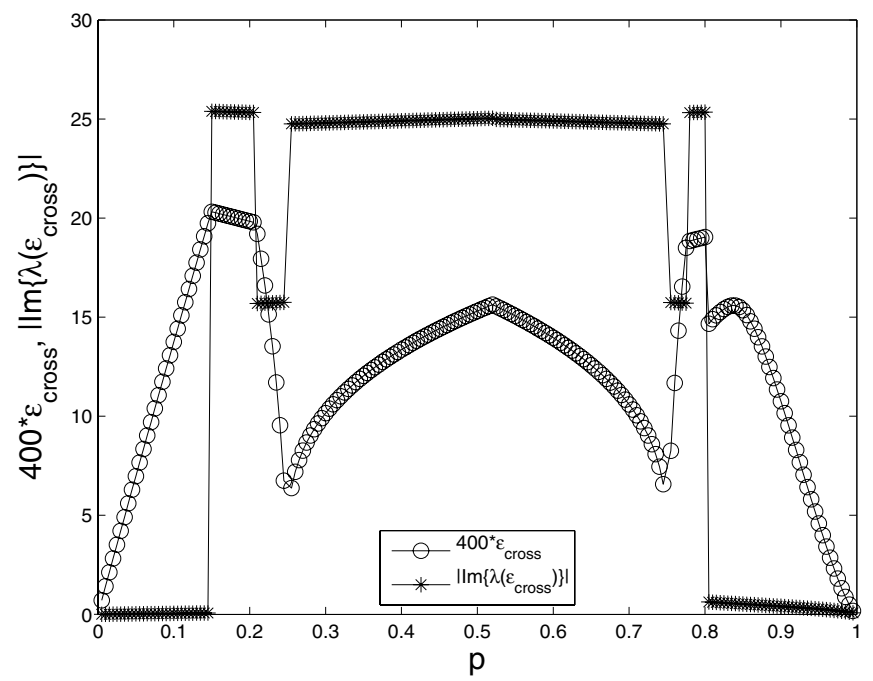

Fig. 7. Combined plot of "first crossing" $\varepsilon_{\text {cross }}$ (multiplied by 400) and the imaginary part of the "first crossing eigenvalue" $\lambda\left(\varepsilon_{\text {cross }}\right)$ (taken in absolute value) versus $p$ for $N_{x}=64, N_{y}=32$, and $N=30$.

reformulate the algorithm in the presence of resonance to address the exceptional values of the Bloch period which our current algorithm excludes.

\section{Appendix A Comments on the Linear Estimate}

In this appendix we comment on some of the key issues involved in establishing Lemma 5.5. As the proof is quite similar to that of the "elliptic estimate" given in the paper of Nicholls and Reitich [NR05], we will not provide all of the details. However, there are some important differences (particularly for a numerical implementation), and we point those out here.

Due to the Bloch boundary conditions, (36e) and (36f), we can expand $v$ and $\zeta$ as

$$
v(x, y)=\sum_{k \in \Gamma^{\prime}} \hat{v}_{k}(y) e^{i(k+p) \cdot x}, \quad \zeta(x)=\sum_{k \in \Gamma^{\prime}} \hat{\zeta}_{k} e^{i(k+p) \cdot x} .
$$

Equations (36) demand that $\left(\hat{v}_{k}, \hat{\zeta}_{k}, \mu\right)$ satisfy, for every $k \in \Gamma^{\prime}$,

$$
\begin{aligned}
& \partial_{y}^{2} \hat{v}_{k}(y)-|k+p|^{2} \hat{v}_{k}(y)=\hat{F}_{k}(y), \quad-a<y<0, \\
& \partial_{y} \hat{v}_{k}(-a)-|k+p| \tanh ((h-a)|k+p|) \hat{v}_{k}(-a)=\hat{J}_{k}, \\
& {[\lambda+i c \cdot(k+p)] \hat{\zeta}_{k}-\partial_{y} \hat{v}_{k}(0)=\hat{Q}_{k}-\delta_{k, \kappa} \mu q_{\kappa},} \\
& {[\lambda+i c \cdot(k+p)] \hat{v}_{k}(0)+\left[g+\sigma|k+p|^{2}\right] \hat{\zeta}_{k}=\hat{R}_{k}-\delta_{k, \kappa} \mu r_{\kappa} .}
\end{aligned}
$$


As long as $(k+p) \neq 0$, the solution of (43a) and (43b) can be written

$$
\begin{aligned}
\hat{v}_{k}(y)= & C_{k}\left\{\beta_{k} e^{|k+p| y}+\alpha_{k} e^{-|k+p| y}\right\}-\left\{\frac{\hat{J}_{k}}{|k+p| \beta_{k}}+\frac{\tilde{\alpha}_{k} T_{2}(-a)}{2|k+p| \beta_{k}}\right\} e^{-|k+p| y} \\
& -\frac{1}{2|k+p|}\left\{T_{1}(y)+T_{2}(y)\right\},
\end{aligned}
$$

where

$$
\begin{aligned}
& T_{1}(y)=T_{1}[F](y):=\int_{-a}^{y} e^{|k+p|(s-y)} F(s) d s, \\
& T_{2}(y)=T_{2}[F](y):=\int_{y}^{0} e^{|k+p|(y-s)} F(s) d s, \\
& \tilde{\alpha}_{k}:=(1-\tanh ((h-a))|k+p|) .
\end{aligned}
$$

Using these, we can resolve (43c) and (43d) into

$$
M_{k} w_{k}=b_{k}, \quad b_{k}:=\left(\tilde{Q}_{k}, \tilde{R}_{k}\right)^{T},
$$

cf. (25), where $M_{k}$ and $w_{k}$ are defined as

$$
M_{k}:=\left(\begin{array}{cc}
\lambda+i c \cdot(k+p) & -|k+p|\left(\beta_{k}-\alpha_{k}\right) \\
g+\sigma|k+p|^{2} & (\lambda+i c \cdot(k+p))\left(\beta_{k}+\alpha_{k}\right)
\end{array}\right), \quad w_{k}:=\left(\begin{array}{l}
\hat{\zeta}_{k} \\
C_{k}
\end{array}\right),
$$

cf. (26), and

$$
\begin{aligned}
\tilde{Q}_{k} & :=\hat{Q}_{k}-\delta_{k, \kappa} \mu q_{\kappa}-\Phi_{k}, \\
\tilde{R}_{k} & :=\hat{R}_{k}-\delta_{k, \kappa} \mu r_{\kappa}+\frac{(\lambda+i c \cdot(k+p))}{|k+p|} \Phi_{k},
\end{aligned}
$$

where

$$
\Phi_{k}:=\frac{\hat{J}_{k}}{\beta_{k}}+\frac{\tilde{\alpha}_{k} T_{2}(-a)}{2 \beta_{k}}+\frac{T_{1}(0)}{2} .
$$

By our condition of nonresonance (31), when $k \neq \kappa$, the determinant function

$\Lambda(k, p ; \lambda, c, g, h, \sigma)=(\lambda+i c \cdot(k+p))^{2}+\left(g+\sigma|k+p|^{2}\right)|k+p| \tanh (h|k+p|)$,

cf. (27), is nonzero, and (44) is uniquely solvable; the resulting $\left(\zeta_{k}, C_{k}\right)$ can be used to establish the elliptic estimates (39), cf. [NR05].

In the case $k=\kappa$ (where $\kappa$ has been chosen so that $\Lambda(\kappa, p)=0$ ), the system (44) is, of course, generally not uniquely solvable. However, since $\mu$ is yet to be determined, we can use it, as we demonstrate below, to enforce the existence of a solution. Once this is accomplished we will make an orthogonality demand that will deliver a unique solution.

Regarding existence of solutions, it is easy to show that in order for $b_{\kappa}$ to be in the range of $M_{\kappa}$ the following condition must be satisfied:

$$
(\lambda+i c \cdot(\kappa+p))\left(\beta_{\kappa}+\alpha_{\kappa}\right) \tilde{Q}_{\kappa}+|\kappa+p|\left(\beta_{\kappa}-\alpha_{\kappa}\right) \tilde{R}_{\kappa}=0 .
$$


Simplifying, we find that this is true if $\mu=n_{\kappa} / d_{\kappa}$, where

$$
\begin{aligned}
n_{\kappa}:= & (\lambda+i c \cdot(\kappa+p))\left(\beta_{\kappa}+\alpha_{\kappa}\right)\left\{\hat{Q}_{\kappa}+\Phi_{\kappa}\right\}, \\
& +|\kappa+p|\left(\beta_{\kappa}-\alpha_{\kappa}\right)\left\{\hat{R}_{\kappa}+\frac{(\lambda+i c \cdot(\kappa+p))}{|\kappa+p|} \Phi_{\kappa}\right\}, \\
d_{\kappa}:= & (\lambda+i c \cdot(\kappa+p))\left(\beta_{\kappa}+\alpha_{\kappa}\right) q_{\kappa}+|\kappa+p|\left(\beta_{\kappa}-\alpha_{\kappa}\right) r_{\kappa} .
\end{aligned}
$$

The choices of $q_{\kappa}$ and $r_{\kappa}$ given in (37) are meant to represent the Fourier coefficients of $\zeta_{0}(x)$ and $v_{0}(x, 0)$ - see (30) — and we can use them to further simplify $d_{\kappa}$ to

$$
d_{\kappa}=2 \rho(\lambda+i c \cdot(\kappa+p))|\kappa+p|\left(\beta_{\kappa}^{2}-\alpha_{\kappa}^{2}\right)
$$

which can be shown to be nonzero. Now, to specify a unique solution, we use (38), i.e. $\hat{\zeta}_{\kappa}=0$, so that $\zeta$ is orthogonal to $\zeta_{0}(x)=q_{\kappa} \exp (i(\kappa+p) \cdot x)$. Other choices can, of course, be made (e.g. the Schwartz parameterization [Sch74]); however, we have found this simple orthogonalization procedure to be quite effective.

\section{Acknowledgments}

The author gratefully acknowledges support from the NSF through grant No. DMS0537511 .

\section{References}

[Ada75] R. A. Adams. Sobolev spaces. Pure and Applied Mathematics, vol. 65. Academic Press [A subsidiary of Harcourt Brace Jovanovich, Publishers], New York-London, 1975.

[BF67] T. B. Benjamin and J. E. Fier. The disintegration of wave trains on deep water. Part I: Theory. J. Fluid Mech., 27:417-430, 1967.

[BGM96] G. A. Baker Jr. and P. Graves-Morris. Padé approximants. Cambridge University Press, Cambridge, second edition, 1996.

[CHQZ88] C. Canuto, M. Yousuff Hussaini, A. Quarteroni, and T. A. Zang. Spectral methods in fluid dynamics. Springer-Verlag, New York, 1988.

[CMR80] J. Chandezon, D. Maystre, and G. Raoult. A new theoretical method for diffraction gratings and its numerical application. J. Optics, 11(4):235-241, 1980.

[CNO0] W. Craig and D. P. Nicholls. Traveling two- and three-dimensional capillary gravity water waves. SIAM J. Math. Anal., 32(2):323-359, 2000.

[CS85] B. Chen and P. G. Saffman. Three-dimensional stability and bifurcation of capillary and gravity waves on deep water. Stud. Appl. Math., 72:125-147, 1985.

[DK99] F. Dias and C. Kharif. Nonlinear gravity and capillary-gravity waves. In Annual review of fluid mechanics, Vol. 31, pages 301-346. Annual Reviews, Palo Alto, CA, 1999.

[GO77] D. Gottlieb and S. A. Orszag. Numerical analysis of spectral methods: Theory and applications. CBMS-NSF Regional Conference Series in Applied Mathematics, no. 26. Society for Industrial and Applied Mathematics, Philadelphia, PA, 1977.

[GVL96] G. H. Golub and C. F. Van Loan. Matrix computations. Johns Hopkins Studies in the Mathematical Sciences. Johns Hopkins University Press, Baltimore, MD, third edition, 1996. 
[HN05] B. Hu and D. P. Nicholls. Analyticity of Dirichlet-Neumann operators on Hölder and Lipschitz domains. SIAM J. Math. Anal., 37(1):302-320, 2005.

[IKR99] M. Ioualalen, C. Kharif, and A. Roberts. Stability regimes of finite depth shortcrested water waves. J. Phys. Oceanogr., 29(9):2318-2331, 1999.

[IO02] M. Ioualalen and M. Okamura. Structure of the instability associated with harmonic resonance of short-crested waves. J. Phys. Oceanogr., 32(5):1331-1337, 2002.

[Lam93] H. Lamb. Hydrodynamics. Cambridge University Press, Cambridge, sixth edition, 1993.

[LCGP99] L. Li, J. Chandezon, G. Granet, and J. P. Plumey. Rigorous and efficient gratinganalysis method made easy for optical engineers. Appl. Opt., 38(2):304-313, 1999.

[LH78a] M. S. Longuet-Higgins. The instabilities of gravity waves of finite amplitude in deep water. I: Superharmonics. Proc. Roy. Soc. London Ser. A, 360(1703):471-488, 1978.

[LH78b] M. S. Longuet-Higgins. The instabilities of gravity waves of finite amplitude in deep water. II: Subharmonics. Proc. Roy. Soc. London Ser. A, 360(1703):489-505, 1978.

[Mie97] A. Mielke. Instability and stability of rolls in the Swift-Hohenberg equation. Commun. Math. Phys., 189(3):829-853, 1997.

[MS86] R. S. MacKay and P. G. Saffman. Stability of water waves. Proc. Roy. Soc. Lond. A, 406:115-125, 1986.

[NR01a] D. P. Nicholls and F. Reitich. A new approach to analyticity of Dirichlet-Neumann operators. Proc. Roy. Soc. Edinburgh Sect. A, 131(6):1411-1433, 2001.

[NR01b] D. P. Nicholls and F. Reitich. Stability of high-order perturbative methods for the computation of Dirichlet-Neumann operators. J. Comput. Phys., 170(1):276-298, 2001.

[NR03] D. P. Nicholls and F. Reitich. Analytic continuation of Dirichlet-Neumann operators. Numer. Math., 94(1):107-146, 2003.

[NR04a] D. P. Nicholls and F. Reitich. Shape deformations in rough surface scattering: Cancellations, conditioning, and convergence. J. Opt. Soc. Am. A, 21(4):590-605, 2004.

[NR04b] D. P. Nicholls and F. Reitich. Shape deformations in rough surface scattering: Improved algorithms. J. Opt. Soc. Am. A, 21(4):606-621, 2004.

[NR05] D. P. Nicholls and F. Reitich. On analyticity of traveling water waves. Proc. Roy. Soc. Lond. A, 461(2057):1283-1309, 2005.

[NR06] D. P. Nicholls and F. Reitich. Rapid, stable, high-order computation of traveling water waves in three dimensions. Eur. J. Mech. B Fluids, 25(4):406-424, 2006.

[NT06] D. P. Nicholls and M. Taber. Joint analyticity and analytic continuation for DirichletNeumann operators on doubly perturbed domains. J. Math. Fluid Mech. (to appear), 2006.

[Phi57] N. A. Phillips. A coordinate system having some special advantages for numerical forecasting. J. Atmospheric Sci., 14(2):184-185, 1957.

[RS78] M. Reed and B. Simon. Methods of modern mathematical physics. IV: Analysis of operators. Academic Press [Harcourt Brace Jovanovich Publishers], New York, 1978.

[RSh81] J. Reeder and M. Shinbrot. Three-dimensional nonlinear wave interaction in water of constant depth. Nonlin. Anal., 5:303-323, 1981.

[Sch74] L. W. Schwartz. Computer extension and analytic continuation of Stokes' expansion for gravity waves. J. Fluid Mech., 62:553-578, 1974.

[Zak68] Vladimir Zakharov. Stability of periodic waves of finite amplitude on the surface of a deep fluid. J. Appl. Mech. \& Tech. Phys., 9:190-194, 1968. 